\title{
Effective Quantum Observables
}

\section{Baaz ${ }^{1}$, N. Brunner ${ }^{2}$ and K. Svozil ${ }^{3}$}

\begin{abstract}
Thought experiments about the physical nature of set theoretical counterexamples to the axiom of choice motivate the investigation of peculiar constructions, e.g. an infinite dimensional Hilbert space with a modular quantum logic. Applying a concept due to BENIOFF, we identify the intrinsically effective Hamiltonians with those observables of quantum theory which may coexist with a failure of the axiom of choice. Here a self adjoint operator is intrinsically effective, iff the Schrödinger equation of its generated semigroup is soluble by means of eigenfunction series expansions.
\end{abstract}

\section{Introduction and Summary}

0.1. The problem. The formalism of quantum theory is known to depend in an essential way on infinite structures, whence one might wonder about the effects of infinity. A proper analysis of this problem seems to require the reconstruction of quantum theory within some system of constructive mathematics.

Hellmann [He93] has questioned the feasibility of the standard approach towards constructive quantum theory by means of computability theory. Instead we investigate effective quantum theory which rest on a very mild condition of constructivity. A concept is effective in the sense of SiERPINSKI, if it does not require the axiom of choice. Nevertheless, even the very notion of a self adjoint operator as an observable of quantum theory may become meaningless in this context. This motivates the question, if there is an alternative definition for observables which does not depend on the axiom of choice $(\mathrm{AC})$, and if so, what is its relation to the usual one.

0.2. A solution. Our point of departure is the Kochen and Specker [Ko67] definition of a quantum theoretical observable on a finite dimensional Hilbert

\footnotetext{
1 Abt. Theoretische Informatik, TU Wien, Wiedner Hauptstraße 8-10, A-1040 Wien

2 Inst. Math., U. Bodenkultur, Gregor Mendel-Str.33, A-1180 Wien

3 Inst. Theoretische Physik, TU Wien, Wiedner Hauptstraße 8-10, A-1040 Wien
} 
space. We apply general requirements about the notion of measurement in empirical structures in order to extend this definition to infinite dimensional spaces. The definition of these finitary observables does not depend on $A C$. Not surprisingly then, finitary observables do not correspond to self adjoint operators but to a different class of finitary mappings on Hilbert space. There is, however, a class of quantum like finitary mappings which have the same functional calculus as the quantum theoretical observables.

While in the presence of $A C$, these quantum like observables reduce essentially to the finite dimensional observables of quantum theory, there are permutation models of $Z F A$ set theory minus $A C$ which admit nontrivial quantum like observables. $Z F A$ is a variant of $Z F$ set theory which permits a set $A$ of atoms (objects without elements; c.f. JECH [Je73]). Investigations into the physical nature of RUSSELL's socks provide even a physical motivation for these models. Moreover, the quantum like observables in such models may be extended to self adjoint operators of the surrounding world $Z F C(=Z F+A C)$ in a way which respects the functional calculus. Our proposed constructive variant of quantum theory is the $Z F C$ theory of these intrinsically effective self adjoint operators.

Implicitly, intrinsically effective Hamiltonians have been considered in thought experiments of a quantum philosophical nature. For example in 1987, in the context of speculations about quantum chaos, A. PEREs (c.f. [Pe93]) has determined as non-chaotic those Hamiltonians, whose Schrödinger equations may be solved by means of eigenfunction series expansions. The latter property characterizes intrinsic effectivity (lemma 16.)

0.3. Acknowledgements. Preliminary versions of our paper have been presented in June 1992 at a conference of the DVMLGdM at Münster and in March 1993 at a meeting of the contact group in mathematical logic at Louvaine de la Neufe. The discussions at these meetings have helped to improve the presentation of the present paper.

Thanks for her help with the preparation of the manuscript are due to Mrs. Holy (U. BoKu.). The authors gratefully remember helpful comments by professors Ruppert (U. BoKu.), HeJtmaneK and Schachermayer (University of Vienna).

\section{A general notion of observables}

1.1. Observations. A measurement is an assignment of mathematical constructs to the elements of an empirical structure. Any particular measurement may be represented by the data which it produces. Thus if $X$ is the empirical structure in question, $Y$ is the set of intended values and $E \subseteq X$ is the 
set of states which are relevant for the measurement situation, then the set $f=\{(x, f x): x \in E\}$ of all collected data forms a function $f: E \rightarrow Y$ which describes the measurement in an extensional way.

We let $\mathcal{F}(X, Y)$ be the set of all partial functions from $X$ to $Y$ and represent all possible measurements by a set $\mathcal{A} \subseteq \mathcal{F}(X, Y)$ of allowable functions.

1.2. Information. The measurements are quasiordered according to their information content by a reflexive and transitive relation $\leq$ on $\mathcal{A}$. If $f \subseteq g \in \mathcal{A}$ (set inclusion), then we require $f \in \mathcal{A}$ and $f \leq g$, since $f$ can be obtained from $g$ by forgetting data. The quasiordering reflects the predictions which can be drawn from an empirical theory.

1.3. Knowledge. An observable $\mathcal{O} \subseteq \mathcal{A}$ shall be a nonempty collection of measurements which represents a state of knowledge corresponding to measurements from the same class of procedures. The following conditions seem natural: (i) If $f \leq g \in \mathcal{O}$, then $f \in \mathcal{O}$, since $f$ contains less information. (ii) If $f \in \mathcal{O}$ and $g \in \mathcal{O}$, then for some $h \in \mathcal{O}$ both $f \leq h$ and $g \leq h ; h$ corresponds to an increased amount of information by the repeated application of the same experimental scheme. Thus $\mathcal{O}$ is an order ideal in $\mathcal{A}$.

The ideal $\mathcal{O}=\{\varnothing\}$ corresponds to "no empirical evidence". Observables are idealizations which represent maximal knowledge. We arrive at the following definition.

An observable $\mathcal{O}$ for an empirical structure $(\mathcal{A}, \leq)$ is a maximal order ideal in $\mathcal{A}$.

1.4. Functions. An important feature of observables is the existence of an functional calculus resembling that on $Y$.

If $F: Y^{n} \rightarrow Y$ and $f_{1}, \cdots f_{n} \in Y^{E}$ are functions, then $F \circ\left(f_{1}, \cdots f_{n}\right) \in$ $Y^{E}$ is defined as $F_{\circ}\left(f_{1}, \cdots f_{n}\right)(a)=F\left(f_{1}(a), \cdots f_{n}(a)\right)$. For $F$ we assume, that $F_{\circ}\left(f_{1}, \cdots f_{n}\right) \in \mathcal{A}$ for all $f_{i} \in \mathcal{A}$ with the same domain and that $F_{\circ}\left(f_{1}, \cdots f_{n}\right) \leq$ $F \circ\left(g_{1}, \cdots g_{n}\right)$ for all $f_{i} \in \mathcal{A} \cap Y^{E}, g_{i} \in \mathcal{A} \cap Y^{G}$ such that $f_{i} \leq g_{i}$ for all $i$.

Ideals $\mathcal{O}_{1}, \cdots \mathcal{O}_{n}$ are commeasurable, if for each sequence $f_{i} \in \mathcal{O}_{i}$ of functions there exist $g_{i} \in \mathcal{O}_{i}$ such that $g_{i} \geq f_{i}$ and all functions $g_{i}$ have the same domain. With $F$ we associate a function $\widehat{F}$ which is defined on commeasurable ideals $\mathcal{O}_{1}, \cdots \mathcal{O}_{n}: \widehat{F}\left(\mathcal{O}_{1}, \cdots \mathcal{O}_{n}\right)=\left\{f \in \mathcal{A}: f \leq F_{\circ}\left(g_{1}, \cdots g_{n}\right)\right.$ for some sequence $g_{i} \in \mathcal{O}_{i}$ of functions with the same domain $\}$. This is an ideal and $\mathcal{O}_{1}, \cdots \mathcal{O}_{n}, \widehat{F}\left(\mathcal{O}_{1}, \cdots \mathcal{O}_{n}\right)$ are commeasurable. However, even if all $\mathcal{O}_{i}$ are observables, $\widehat{F}\left(\mathcal{O}_{1}, \cdots \mathcal{O}_{n}\right)$ may fail to be maximal.

A function $F: Y^{n} \rightarrow Y$ is admissible, if in addition to the requirements for the definition of $\widehat{F}, \widehat{F}$ maps commeasurable observables to observables. In section 1.5 there is a counterexample which shows, that the set of admissible functions in general is not closed under composition. For observables of quantum 
theory, commeasurability coincides with commensurability (c.f. [Ko67]) and the functional calculus is the usual one.

1.5. Counterexample. We illustrate our terminology by a simple notion of measurement, where names $y \in Y$ are attributed to the objects $x \in X$ and renaming or the identification of names decreases the information content. To this end we consider the structure $(\mathcal{A}, \leq)$, where $\mathcal{A}=\{f \in \mathcal{F}(X, Y)$ : dom $f$ is finite $\}$ and $f \leq g$, iff $\operatorname{dom} f \subseteq \operatorname{dom} g$ and there is a function $h: Y \rightarrow Y$ such that $f=h \circ g \mid \operatorname{dom} f$ [that is, $\forall x \in \operatorname{dom} f: f(x)=h(g(x))$.]

Lemma 1. In $Z F A$, if $Y$ is infinite, then the set $(\mathcal{I}, \subseteq)$ of all order ideals of $(\mathcal{A}, \leq)$ is isomorphic to the complete lattice $(\Pi, \succeq)$ of all partitions $\mathcal{P}$ of $X^{+}=X \cup\{a\}, a \notin X$ an additional distinguished object, where $\mathcal{P} \preceq \mathcal{Q}$, iff $\mathcal{P}$ is a refinement of $\mathcal{Q}$.

Proof. If $\mathcal{P}$ is a partition of $X^{+}$, we define $j(\mathcal{P}) \subseteq \mathcal{A}$ as $j(\mathcal{P})=\{f \in$ $\mathcal{A}$ : dom $f \cap P=\varnothing$, if $a \in P \in \mathcal{P}$, and $f x=f y$, if $\{x, y\} \subseteq P$ for some $P \in \mathcal{P}\}$. Obviously, $\varnothing \in j \mathcal{P}$ and $f \leq g \in j(\mathcal{P})$ implies $f \in j(\mathcal{P})$. If $f$ and $g$ are in $j(\mathcal{P})$ and $E=\operatorname{dom} f \cup \operatorname{dom} g$ intersects exactly $n$ elements $P_{1}, \cdots P_{n}$ of $\mathcal{P}$, we let $y_{1}, \cdots y_{n}$ be arbitrary $n$ elements of $Y$ and define $h: E \rightarrow Y$ as $h(x)=y_{i}$, if $x \in P_{i}$. Then $h \in j(\mathcal{P})$ and $h \geq f, h \geq g$, whence $j(\mathcal{P})$ is an order ideal.

If $\mathcal{O} \subseteq \mathcal{A}$ is an order ideal, we define the following relation $\sim$ on $X^{+}: \operatorname{dom} \mathcal{O}=\cup\{\operatorname{dom} f: f \in \mathcal{O}\}$ and $x \sim y$, iff $\{x, y\} \cap \operatorname{dom} \mathcal{O}=\varnothing$ or $\{x, y\} \subseteq \operatorname{dom} \mathcal{O}$ and $f x=f y$ for all $f \in \mathcal{O}$ such that $\{x, y\} \subseteq \operatorname{dom} f$. Note that, if $E \subseteq \operatorname{dom} \mathcal{O}$ is finite, then there is a function $f_{E} \in \mathcal{O}$ such that $E \subseteq \operatorname{dom} f_{E}$ (if $x \in \operatorname{dom} f_{x}$ and $f_{x} \in \mathcal{O}$ for $x \in E$, then let $f_{E} \in \mathcal{O}$ be an upper bound of these finitely many $f_{x}$ ). This defines an equivalence relation, for if $x \sim y, y \sim z$ and $\{x, y, z\} \subseteq \operatorname{dom} \mathcal{O}$, but not $x \sim z$, then for some $f \in \mathcal{O} \operatorname{dom} f \supseteq\{x, y, z\}$ and for some $g \in \mathcal{O} g x \neq g z$. If $h \in \mathcal{O}$ and $h \geq f, g$, then $h x \neq h z$ and $\operatorname{dom} h \supseteq\{x, y, z\}$. It follows, that $h x \neq h y$ or $h y \neq h z$, a contradiction, whence $\sim$ is transitive. We let $\pi \mathcal{O}$ be the associated partition of $X^{+}$.

One easily verifies, that $\pi \mathcal{O} \preceq \pi \mathcal{O}^{\prime}$, if $\mathcal{O} \supseteq \mathcal{O}^{\prime}$, and $j \mathcal{P} \subseteq j \mathcal{P}^{\prime}$, if $\mathcal{P}^{\prime} \preceq \mathcal{P}$. Moreover $\pi j \mathcal{P}=\mathcal{P}$ for all partitions $\mathcal{P}$ and $j \pi \mathcal{O}=\mathcal{O}$ for all order ideals $\mathcal{O}$. Concerning the latter identity, $\mathcal{O} \subseteq j \pi \mathcal{O}$ is obvious. If conversely $f: E \rightarrow Y$ is in $j \pi \mathcal{O}$, then $E \subseteq \operatorname{dom} \mathcal{O}$ and moreover $f x \neq f y$ implies $g_{x y}(x) \neq g_{x y}(y)$ for some $g_{x y} \in \mathcal{O}$. We let $g \in \mathcal{O}$ be an upper bound of $f_{E}$ and all $g_{x y}$. Then $g \geq f$ and therefore $f \in \mathcal{O}$.

\section{e. o. p.}

Since $\mathcal{A}$ is an order ideal, it is the only observable. As follows from lemma 1, if $X$ and $Y$ are infinite, then $\mathcal{O}$ is an observable, iff for each finite set of objects there exists a measurement in $\mathcal{O}$ which assigns different names to these objects. Here the cardinality of $Y$ needs not be larger than that of $X$. Therefore a mapping $f: Y \rightarrow Y, Y$ infinite, is admissible for the functional calculus, iff its range is infinite. [If the range of $f$ is finite, then $\widehat{f} \mathcal{A}$ is not an ideal, while if the range of $f$ is infinite, then $\widehat{f} \mathcal{A}=\mathcal{A}$.] The family of these mappings is not closed under composition. 
An order ideal $\mathcal{O}$ is scalable, if there exists a function $f: X \rightarrow Y$ such that $\mathcal{O}=\mathcal{O}(f)$, where $\mathcal{O}(f)=\{g \in \mathcal{A}: g \leq f \mid E$ for some finite $E \subseteq X\}$. Since $\pi \mathcal{O}(f)=\left\{\{a\}, f^{-1^{\prime \prime}}\{y\}: y \in f^{\prime \prime} X\right\}, \mathcal{A}$ is scalable, iff there exists an injective function $f: X \rightarrow Y$, a so called cardinal scale. This notion is taken from mathematical psychology. In $Z F A, \mathcal{O}(f) \supseteq \mathcal{O}(g)$, iff there is a function $h: Y \rightarrow Y$ such that $g=h_{\circ} f$ (c.f. Hindman and Milnes [Hi84] for related topological conditions.) In general $\widehat{h} \mathcal{O}(f) \neq \mathcal{O}(h \circ f)$. [If $f(x) \neq f(y)$ and $h_{\circ} f(x)=h_{\circ} f(y)$ and the range of $h$ is infinite, then $\widehat{h} \mathcal{O}(f)=\mathcal{O}(f) \neq \mathcal{O}\left(h_{\circ} f\right)$.]

The partition principle is the assertion, that the injective and the surjective orderings of cardinals coincide. It is known to imply the wellorderable axiom of choice (D. PINCUS in [Pe78], p. 587), but it is still an open question, if it is equivalent to $A C$. As follows from lemma 2 below, in $Z F$ set theory the partition principle is equivalent to the assertion, that for all structures $(\mathcal{A}, \leq)$ with infinite sets of names the set of all scalable ideals is closed under bounded joins of order ideals.

Lemma 2. In $Z F$, if $Y$ is infinite, then the join of a nonempty family of scalable ideals which are bounded from above by a scalable ideal is again scalable, iff the following holds: If $\mu \leq_{*} \kappa \leq_{*}|X|$ and $\kappa \leq|Y|$, then $\mu \leq|Y|$.

Proof. $|X|$ is the ScotT cardinality of $X .|X| \leq|Y|$, if there exists an injective $f: X \rightarrow Y$ and $|X| \leq_{*}|Y|$, if there exists a surjective $f: Y \rightarrow X$.

The representable ideals are order isomorphic to $\left(\Pi^{\prime}, \succeq\right), \Pi^{\prime}$ the set of all partitions $\mathcal{P}^{\prime}$ of $X$ such that $\left|\mathcal{P}^{\prime}\right| \leq|Y|\left(\pi^{\prime}(\mathcal{O})=\pi(\mathcal{O}) \backslash\{\{a\}\}\right.$ defines the isomorphism). Since for a partition $\mathcal{P}$ of $X \quad|\mathcal{P}| \leq_{*}|X|$ and for partitions $\mathcal{P} \preceq \mathcal{Q} \quad|\mathcal{P}| \geq_{*}|\mathcal{Q}|$, the assumptions on the cardinals imply the Dedekindcompleteness of the partially ordered set $\Pi^{\prime}$. For if $\mathcal{L} \preceq \mathcal{P}$ for all $\mathcal{P} \in \Psi \subseteq \Pi^{\prime}$ and some $\mathcal{L} \in \Pi^{\prime}$, then $\mathcal{L} \preceq \inf \Psi$ (formed in the set of all partitions of $X$ ) and $|\inf \Psi| \leq_{*}|\mathcal{L}| \leq_{*}|X|$, whence $\inf \Psi \in \Pi^{\prime}$.

If conversely $\mu \leq_{*} \kappa \leq_{*}|X|$ and $\kappa \leq|Y|, \mu=|M|$ and $\kappa=|K|$, then a surjective mapping $f: X \rightarrow K$ defines a partition $\mathcal{P}=\left\{f^{-1^{\prime \prime}}\{y\}: y \in f^{\prime \prime} X \subseteq\right.$ $K\} \in \Pi^{\prime}$ such that there is a surjective $g: \mathcal{P} \rightarrow M$. We set $\mathcal{Q}=\left\{\cup g^{-1^{\prime \prime}}\{m\}: m \in\right.$ $M$ \}, a partition of $X$ of cardinality $\mu$. Since $\mathcal{Q}=\inf \Psi$ in the set of all partitions of $X$, where $\Psi=\left\{\left\{\cup g^{-1^{\prime \prime}}\{m\}, \cup g^{-1^{\prime \prime}}(M \backslash\{m\})\right\}: m \in M\right\} \subseteq \Pi^{\prime}$, and because $\mathcal{P} \preceq \mathcal{L}$ for all $\mathcal{L} \in \Psi, \mathcal{Q}=\inf \Psi \in \Pi^{\prime}$, if $\Pi^{\prime}$ is closed under bounded infima. In this case $\mu=|\mathcal{Q}| \leq|Y|$.

e. o. p.

There is a similar theory of preference structures. In the definition of $(\mathcal{A}, \leq)$ we let $(Y,<)$ be an infinite dense linear order without endpoints and $f \leq g$, iff $f=h \circ g \mid \operatorname{dom} f$, where $h: Y \rightarrow Y$ is nondecreasing. Then as in lemma 1 the observables on $X$ correspond to the linear orderings on $X$. Hence the ordering principle (each set is orderable) is equivalent with the assertion, that each set admits observables. Observables are commeasurable, iff their induced orderings coincide. 


\section{An effective version of quantum theory}

2.1. Finitary quantum mechanics. We consider the finitary quantum structure $\mathcal{A}=\{f \in \mathcal{F}(H, \mathbb{R})$ : dom $f$ a finite $O N$ system $\}$ and $f \leq g$, iff $\widetilde{f} \subseteq \widetilde{g}$, where $\tilde{f}$ : span dom $f \rightarrow H$ is given by

$$
\tilde{f}(x)=\sum_{a \in \operatorname{dom} f} f(a) \cdot<x, a>\cdot a
$$

Here $H$ is a complex Hilbert space (completeness is defined in terms of Cauchy sequences) of pure states with the scalar product $\langle\cdot, \cdot\rangle$. The finitary observables on $H$ are the observables for this structure.

If $H$ is finite dimensional, then this notion is equivalent with the definition of the observables of quantum theory in Kochen and SPECKER [Ko65]. In general, this is no longer true.

2.2. Functional calculus. As has been pointed out by Kochen and SPECKER [Ko67], in order to correspond to a quantum theoretical observable, a finitary observable needs to obey the same functional calculus. We now identify these quantum like observables with a class of linear mappings. Symmetric linear mappings which satisfy the condition of the following lemma are finitary.

Lemma 3. In $Z F A$ the set $(\mathcal{I}, \subseteq)$ of the ideals of $(\mathcal{A}, \leq)$ is isomorphic with the set $(\mathcal{S}, \subseteq)$ of all symmetric linear operators $A$ : $\operatorname{dom} A \rightarrow H$, where $\operatorname{dom} A$ is the linear span (not necessarily closed) of the set $E V(A)$ of all eigenvectors of $A$.

Proof. If $\mathcal{O} \subseteq \mathcal{A}$ is an ideal, we set $\mu(\mathcal{O})=\cup\{\tilde{f}: f \in \mathcal{O}\}$. Since $\mathcal{O}$ is directed, $\mu(\mathcal{O})$ is a function and if $E \subseteq \operatorname{dom} \mu(\mathcal{O})$ is finite, then $E \subseteq \operatorname{dom} \tilde{f}$ for some $f \in \mathcal{O}$, whence $\mu(\mathcal{O})$ is linear and symmetric. By definition dom $\tilde{f}=$ span $E V(\widetilde{f})$ and since $E V(\widetilde{f}) \subseteq E V(\mu(\mathcal{O}))$, dom $\mu(\mathcal{O})=\operatorname{span} E V(\mu(\mathcal{O}))$, i.e. $\mu(\mathcal{O}) \in \mathcal{S}$.

If $A \in \mathcal{S}$ and $E \subseteq E V(A)$ is a finite $O N$ system, then we define $A_{E}: E \rightarrow$ $\mathbb{C}$ through $A_{E}(a) \cdot a=A(a), a \in E$. Since the eigenvalues of symmetric matrices are real, $A_{E} \in \mathcal{A}$. We set $j(A)=\left\{f \in \mathcal{A}: f \leq A_{E}\right.$ for some finite $O N$ system $E \subseteq E V(A)\}$ and observe, that $j(A)$ is an order ideal. For if $E, F$ are finite $O N$ systems in $E V(A)$, then $A_{E}, A_{F} \leq A_{G}$, where $G \subseteq E V(A \mid \operatorname{span}(E \cup F))$ is an $O N$ system such that $\operatorname{span} G=\operatorname{span}(E \cup F)$ which exists by diagonalization.

If $A \in \mathcal{S}$, then $\mu j A=\cup\left\{\widetilde{A}_{E}: E \subseteq E V(A)\right.$ a finite $O N$ system $\}=\cup\{A \mid$ span $E: E \subseteq E V(A)$ a finite set $\}=A$. If $\mathcal{O} \in \mathcal{I}$, then $j \mu \mathcal{O}=\left\{f: f \leq \mu(\mathcal{O})_{E}\right.$ for some finite $O N$ system $E \subseteq E V(\mu(\mathcal{O}))\}$. Since $\mu(\mathcal{O})_{E} \leq g$; whenever $g \in \mathcal{O}$ satisfies dom $\widetilde{g} \supseteq E, j \mu \mathcal{O} \subseteq \mathcal{O}$, while $j \mu \mathcal{O} \supseteq \mathcal{O}$ is obvious.

e. o. p. 
If the finitary mapping $A$ satisfies $\operatorname{dom} A=H$, then $A$ corresponds to a finitary observable. Observables of this kind (and the corresponding maps) are quantum like.

As a motivation for this terminology, we note that one may change the definition of a finitary observable, so that in $Z F C$ the new class of observables includes the usual observables of quantum theory. Let $(\mathcal{A}, \leq)$ consist of the symmetric linear maps, partially ordered by inclusion. Then observables are a direct sum of a self-adjoint operator and a finite number of unbounded operators $+T$ or $-T$, where $T=i(1+S)(1-S)^{-1}, S$ the unilateral shift on $\ell_{2}(\omega)$ (voN Neumann [vN29]).

There is also an analogy with nonstandard analysis. We let $*$ be an $\aleph_{1}$-saturated enlargement and consider $H=\left({ }^{*} \mathbb{C}\right)^{\eta}, \eta \in{ }^{*} \mathbb{N}$ (c.f. $[\mathrm{Al} 86]$ ). $\mathcal{A}$ is the set of all internal partial functions $f: \operatorname{dom} f \rightarrow^{*} \mathbb{R}, \operatorname{dom} f$ a hyperfinite $O N$-system in $H$ and $f \leq g$, iff as in section $2.1 \widetilde{f} \subseteq \widetilde{g}$. Then by the proof of lemma 3 internal observables correspond to symmetric and everywhere defined linear mappings $L$ on $H$. If the norm of $L$ is finite, then $L$ defines a bounded self adjoint mapping $\widehat{L}$ on the nonstandard hull $\widehat{H}$ of $H$. If conversely $A$ is a bounded self adjoint mapping on the standard Hilbert space $K$ and $H$ is Sdense in ${ }^{*} K$ (i.e. each $x \in{ }^{*} K$ is infinitely close to some $y \in H$ ), then for some bounded $L$ on $H$ (i.e. an observable of $\mathcal{A}$ ) $A$ is the restriction of $L$ to the standard elements. Thus bounded quantum theory is obtained in principle by means of the following modifications of its finitary variant:

(i) The reals are enlarged to a hyperreal number field;

(ii) finiteness is weakened to hyperfiniteness.

The philosophical implications of (ii) have been investigated by VopÊNKA [Vo79], in whose view hyperfinite infinity describes "the phenomenon of infinity in accordance with our experience, i.e., as a phenomenon involved in the observation of large, incomprehensible sets". To obtain quantum theory, that phenomenon is added to the finitary framework at the level of the data $f \in \mathcal{A}$. In order to represent such data within an internal theory, the modification (i) is needed, whereby the real numbers are supposed to result from empirically realizable measurements and the hyperreals and nonstandard states are theoretical entities. This folklore interpretation is suggested by ABU'L HUdHAYL's ( $9^{\text {th }}$ century AD) explanation of ZENO's paradox of the arrow (JAMmER [Ja74], p. 259).

Lemma 4. In $Z F A$, if $A: \operatorname{dom} A \rightarrow H$ is a finitary mapping and $K$ is a invariant linear manifold in $\operatorname{dom} A$, then the restriction $B=A / K$ is finitary.

Proof. The condition $\operatorname{dom} A=\operatorname{span} E V(A)$ is equivalent to the assertion, that for all $x \in \operatorname{dom} A$ also $A x \in \operatorname{dom} A$ and $\left\{A^{n} x: n \in \omega\right\}$ is finite dimensional. [ If $x=\sum_{i \in k} x_{i}$ and $A x_{i}=\lambda_{i} x_{i}$, then $A^{n} x=\sum_{i \in k} \lambda_{i}{ }^{n} x_{i}$ and $\operatorname{dim}\left\{A^{n} x: n \in\right.$ $\omega\} \leq k$. Conversely, if $S=\operatorname{span}\left\{A^{n} x: n \in \omega\right\}$ is finite dimensional, then by diagonalization $x=A^{0} x \in S=\operatorname{span} E V(A / S) \subseteq \operatorname{span} E V(A)$.] These conditions are inherited by $A / K$.

e. o. p.

If the invariant manifold $K$ is closed in $\operatorname{dom} A$, then we let $P$ be the 
orthogonal projection onto $K$. In $Z F A, P x$ is the unique $y \in K$ such that $\|x-y\|=\inf \{\|x-z\|: z \in K\} . P x$ exists, if $K$ is Cantor complete (the intersection of a nested sequence of closed sets $C_{n}$ whose diameters $\operatorname{diam} C_{n}$ converge to zero is nonempty.) [In view of the proof in [Du57], p. 248-249, $\lim _{\varepsilon \rightarrow 0} \operatorname{diam} K_{\varepsilon}=0, K_{\varepsilon}=\{y \in K:\|x-y\| \leq \varepsilon+\inf \{\|x-z\|: z \in K\}\}$. Hence $\{P x\}=\cap_{\varepsilon>0} K_{\varepsilon}$.] The existence of orthogonal projections on Hilbert spaces depends on the axiom of choice, if no further completeness assumptions are added (lemma 22.) Therefore some authors (c.f. [Mo91]) require stronger completeness properties in the definition of a Hilbert space. We denote by $K^{\perp}=$ $\{x \in H: x \perp K\}$ the orthogonal complement. Then $A^{\prime \prime}\left(\operatorname{dom} A \cap K^{\perp}\right) \subseteq K^{\perp}[$ pick $x \in K, y \in K^{\perp} \cap \operatorname{dom} A$; then $\left.\langle x, A y\rangle=<A x, y\right\rangle=0$, since $\left.y \perp A x \in K\right]$. If $A x=\lambda x$ then $P x \in E V(A)\left[A(P x)-\lambda(P x)=A(P x-x)-\lambda(P x-x) \in K \cap K^{\perp}.\right]$ Hence $K \cap E V(A)=E V(A / K)=P " E V(A)$.

In $Z F A$, the restriction of a symmetric map $A$ to its domain is closed as a mapping from $\operatorname{dom} A$ to $H$. Hence in $Z F A$ each eigenspace of a finitary map is a closed and invariant subspace of $\operatorname{dom} A$ and moreover, quantum like maps are closed.

Lemma 5. In $Z F A$, the ideals $j\left(A_{i}\right)$ of the finitary mappings $A_{1}, \cdots A_{n}$ are commeasurable, if and only if the mappings $A_{i}$ commute and their domains are equal.

Proof. As follows from the proof of lemma 3 and 1.4., $j\left(A_{i}\right)$ are commeasurable, iff $E V\left(A_{i}\right) \subseteq \operatorname{span} \bigcap\left\{E V\left(A_{i}\right): 1 \leq i \leq n\right\}$. This implies dom $A_{1}=\cdots=$ $\operatorname{dom} A_{n}$ and $A_{i} \cdot A_{j}=A_{j} \cdot A_{i}$. Conversely, let $A \cdot B=B \cdot A$ for $A, B$ finitary and let $\operatorname{dom} A=\operatorname{dom} B$. If $\mu$ is an eigenvalue of $B$ and $K_{\mu}=\operatorname{ker}(B-\mu)$, then $A B=B A$ implies $A^{\prime \prime} K_{\mu} \subseteq K_{\mu}$. We let $C_{\mu}=A / K_{\mu} . C_{\mu}$ is finitary by lemma 4 . Then $K_{\mu}=\operatorname{span} E V\left(C_{\mu}\right)$ and since $E V\left(C_{\mu}\right) \subseteq E V(A) \cap K_{\mu} \subseteq E V(A) \cap E V(B)$ and $D=\operatorname{dom} A=\operatorname{dom} B$ is a direct sum of spaces $K_{\mu}$ it follows, that $\operatorname{span}(E V(A) \cap E V(B))=D$.

\section{e. o. p.}

Commutativity alone does not suffice for commeasurability $[A=0 /\{0\}$ commutes with $B$, iff $B$ is one to one, for $B A=A$ and $A B=0 / \operatorname{ker} B$.]

If $F: \mathbb{R}^{n} \rightarrow \mathbb{R}$ is a function and $j A_{i}$ are commeasurable, then $\widehat{F}\left(j A_{1}, \cdots, j A_{n}\right)$ corresponds to the operator $A=F\left(A_{1}, \cdots, A_{n}\right)$ such that $\operatorname{dom} A=\operatorname{dom} A_{i}$ and for $x \in \bigcap_{i} E V\left(A_{i}\right), A_{i} x=\lambda_{i} x, A x=F\left(\lambda_{1}, \cdots \lambda_{n}\right) \cdot x$.

In $Z F A+$ "Dedekind finite subsets of $\mathbb{R}$ are finite" a function $f: \mathbb{R} \rightarrow$ $\mathbb{R}$ is admissible for the class of all finitary observables on $\ell_{2}(\omega)$, if and only if it is finite to one. [For if $f\left(\lambda_{i}\right)=\lambda, i \in \omega$ and $\lambda_{i}$ a sequence of distinct reals, then the finitary operator $A e_{i}=\lambda_{i} e_{i}, e_{i}$ the canonical $O N$ base of $\ell_{2}(\omega)$, dom $A_{i}=$ span $\left\{e_{i}: i \in \omega\right\}$, corresponds to a finitary observable, but $\widehat{f}(A)=\lambda / \operatorname{dom} A$ is strictly included in the finitary map $\lambda\left(\lambda(x)=\lambda \cdot x, x \in \ell_{2}(\omega)\right)$ and thus $\widehat{f}(A)$ corresponds to an ideal which is not maximal]. In $Z F A$ it follows by the same argument, that if $j A$ is a finitary observable, then $\widehat{f}(A)$ corresponds to a finitary observable for all everywhere defined Borel-functions $f$, iff $\operatorname{dom} A=H$. Hence quantum like observables have the same functional calculus as the observables 
of quantum theory. In $Z F C$ this implies, that $A$ is algebraic, as follows from the closed graph theorem and the following result due to KAPLANSKY [Ka54]: A bounded operator on a Hilbert space $H$ is algebraic, if and only if its finite dimensional invariant subspaces cover $H$.

Lemma 6. In ZFC, a self adjoint operator is finitary, iff it is bounded and algebraic $(p(A)=0$ for some nonzero polynomial $p)$.

Proof. If $A$ is finitary, then $\operatorname{dom} A=\oplus_{\lambda \in \sigma_{p}(A)} \operatorname{ker}(A-\lambda)$ (= linear direct sum of the orthogonal system of eigenspaces) and $\operatorname{im} A \subseteq \operatorname{dom} A$. Since $A$ is self-adjoint, $\operatorname{dom} A$ with the norm $\|x\|_{1}^{2}=\|x\|^{2}+\|A x\|^{2}$ is a Hilbert space (c.f. [Du63], p.1225) and by the closed graph theorem, $A$ is bounded. KaPLANsKY's [Ka54] theorem, applied to $A$ on this space, implies that $A$ is algebraic on dom $A$ and since $\operatorname{dom} A$ is dense for a self adjoint operator $A, A$ extends to an unique bounded [since the spectrum $\sigma(A)$ is finite, $A$ is bounded in the norm of $H$ ] and algebraic operator on $H$. If conversely $A$ is bounded and algebraic, then in view of KAPLANSKY's theorem $H=\operatorname{span}(E V(A))$, whence $A$ is finitary.

e. o. p.

In $Z F C$, quantum like observables are empirically realizable in a weak sense. For as has been shown by ZEILINGER and his collaborators [Re94], the time evolution of algebraic Hamiltonians may be simulated by photon-splitting experiments. [Technically, this result is an analogy of JACOBI's $Q R$-decomposition of a symmetric matrix into a diagonal matrix and two dimensional rotations.]

2.3. Russell's socks. In $Z F C$, there is an abundance of non-effective pure states in an infinitely dimensional Hilbert space. Since KAPLANSKY's theorem depends on $A C$ (BRUNNER [Br86]), these states are responsible for the restrictions on the quantum like observables. We therefore investigate finitary observables in models of $Z F A$ minus the axiom of choice. The most interesting spaces for our purpose are the locally sequentially compact ones (the unit sphere is sequentially compact).

Lemma 7. In ZFA, a Hilbert space $H$ is locally sequentially compact, iff each bounded symmetric operator $A: H \rightarrow H$ is finitary.

Proof. If $H$ is locally sequentially compact, then each $x \in H$ is contained in a finite dimensional invariant subspace $F$ of $A$ (BRUnner [Br86], 4.3). By diagonalization, $F=\operatorname{span}(E V(A \mid F))$, whence $A$ is finitary. If $H$ is not locally sequentially compact, then it contains a copy of $\ell_{2}(\omega)$ (BRUNNER [Br86], 2.1 ). Since $\ell_{2}(\omega)$ is second category, KAPLANSKY's theorem applies, whence no symmetric bounded operator $A$ on $\ell_{2}(\omega)$ with infinite spectrum is finitary. Its extension $A \circ P$ to $H$ ( $P$ the projection onto the Cantor complete subspace $\left.\ell_{2}(\omega)\right)$ is a symmetric bounded operator on $H$ which does not correspond to a finitary observable (c.f. lemma 4).

e. o. p.

Lemma 8. In ZFA, if $A$ is a quantum like map on the Cantor complete Hilbert space $H$ such that each eigenspace of $A$ is finite dimensional, then $H$ is locally sequentially compact. 
Proof. For $\lambda \in \mathbb{R}$ we let $P_{\lambda}$ be the orthogonal projection onto $\operatorname{ker}(A-\lambda)$ and define, for $x \in H$, its support as $s(x)=\left\{\lambda \in \mathbb{R}: P_{\lambda} x \neq 0\right\}$. Then for each sequence $\left\langle x_{n}: n \in \omega\right\rangle$ in $H$ the set $S=\cup\left\{s\left(x_{n}\right): n \in \omega\right\}$ is finite. For otherwise there exists an infinite partition $S_{k} \neq \varnothing, k \in \omega$, of $S$ (an observation due to D. PIncus; c.f. [Br82], corollary 2.2.5.) We set $K_{k}=\mathbf{c l} \oplus_{\lambda \in S_{k}} \operatorname{ker}(A-\lambda)$ and let $Q_{k}$ be the orthogonal projection onto $K_{k}$. Then for each $k \in \omega$ there is a least index $n(k)$ such that $y_{k}=Q_{k} x_{n(k)} \neq 0$, since $Q_{k} \geq P_{\lambda}$ for $\lambda \in S_{k}$. We set $y=\sum_{k \in \omega} \frac{1}{k+1} \cdot \frac{y_{k}}{\left\|y_{k}\right\|}$. Then $y \in H$, but $y \notin \operatorname{span} E V(A)$. For if $y \in \oplus_{i \in n} \operatorname{ker}\left(A-\lambda_{i}\right)$ and $S_{k} \cap\left\{\lambda_{i}: i \in n\right\}=\varnothing$, then $Q_{k} " \oplus_{i \in n} \operatorname{ker}\left(A-\lambda_{i}\right)=\{0\}$, but $Q_{k} y=\frac{1}{k+1} \cdot \frac{y_{k}}{\left\|y_{k}\right\|} \neq 0\left[Q_{k} y_{h}=0\right.$ for $k \neq h$, since distinct eigenspaces of $A$ are orthogonal.] As $y \notin \operatorname{span} E V(A)$ is impossible, if $A$ is quantum like, span $\left\{x_{n}: n \in \omega\right\} \subseteq \oplus_{\lambda \in S} \operatorname{ker}(A-\lambda)$ is finite dimensional, whence $H$ is locally sequentially compact ([Br86], lemma 2.1.)

e. o. p.

There are also set theoretical restrictions on Cantor complete Hilbert spaces $H$ which admit quantum like mappings $A$ whose eigenvalues have finite multiplicities, only. In this case for each infinite $D \subseteq H$ the powerset $\mathcal{P}(D)$ is Dedekind infinite. [For otherwise by the above mentioned result due to PINCus $\{s(x): x \in D\} \subseteq[\mathbb{R}]^{<\omega}$ is finite. Therefore for some $E \in[\mathbb{R}]^{<\omega} B=$ $\{x \in D: s(x)=E\}$ is infinite. But $B$ induces an infinite subset of $\mathbb{C}^{m}$, $m=\sum_{\lambda \in E} \operatorname{dim}(\operatorname{ker}(A-\lambda))$, with a Dedekind finite powerset, contradicting the result of PINCUS.]

The existence of such spaces is motivated by thought experiments about RusSELL's socks, the standard example for a failure of $A C$. In our sense they form a sequence of pairwise disjoint two element sets $P_{n}=\left\{a_{n}, b_{n}\right\}, n \in \omega$, which is a counterexample to the principle of partial dependent choices $[P D C$ : there exists a sequence $F_{k}: C_{n_{k}} \rightarrow P_{n_{k}}$ of functions, where $C_{n_{k}}$ is the set of choice functions on $\left.\left\langle P_{i}: i \in n_{k}\right\rangle, n_{k}<n_{k+1}\right)$ for $\left.k \in \omega\right]$. Thus it is not possible to single out a sock of the $n-t h$ pair in an uniform way, even if one could distinguish the previous socks. We view $\left\{a_{n}, b_{n}\right\}$ as an assembly of identical noninteracting spin $\frac{1}{2}$ particles which obey the FERMI-DiRAC statistics (c.f. JAUCH [Ja68], pp. $249-287)$. Its Hilbert space $H_{n}=\operatorname{span}\left\{e_{1}\left(a_{n}\right) \otimes e_{2}\left(b_{n}\right)-e_{2}\left(a_{n}\right) \otimes e_{1}\left(b_{n}\right)\right\}$ is isomorphic with $L_{n}=\left\{x \in \ell_{2}\left\{a_{n}, b_{n}\right\}: x\left(a_{n}\right)+x\left(b_{n}\right)=0\right\}$. The family of all socks is viewed as the compound system of the distinguishable assemblies. Then the failure of $P D C$ might be considered a set theoretical implementation of the physical independence of these particles. Their Fock space is $F=$ $\oplus_{N \in \omega} \otimes_{n \in N} H_{n}$. Here we may replace $H_{n}$ by $L_{n}$, since the isomorphism does not depend on the ordering of $P_{n}$. The finite tensor product $\otimes_{n \in N} \quad L_{n}$ is $T_{N}$, where

$$
T_{N}=\left\{X \in \ell_{2}\left(C_{N}\right): X(\varphi)=(-1)^{m} X(\psi), \quad \text { if } \quad|\{i \in N: \varphi(i) \neq \psi(i)\}|=m\right\}
$$

and $C_{N}$ is the set of choice functions $\varphi: N \rightarrow \cup_{n \in N} P_{n}, \varphi(n) \in P_{n}$ for $n \in N$. This follows from the definition $\tau_{N}: \Pi_{n \in N} L_{n} \rightarrow T_{N}, \tau_{N}(\mathbf{x})=X$, where $\mathbf{x}=<$ $x_{n}: n \in N>$ and $X(\varphi)=\Pi_{n \in N} x_{n}(\varphi(n))$ is the product in $\mathbb{C}$. The function 
$\tau_{N}$ is a multilinear onto map such that

$$
\begin{aligned}
<\tau_{N}(\mathbf{x}), \tau_{N}(\mathbf{y})>_{T_{N}} & =\sum_{\varphi \in C_{N}} \prod_{n \in N} x_{n}(\varphi(n)) y_{n}(\varphi(n))^{-} \\
& =2^{N} \cdot \prod_{n \in N} x_{n}\left(a_{n}\right) y_{n}\left(a_{n}\right)^{-} \\
& =\prod_{n \in N}<x_{n}, y_{n}>_{L_{n}} .
\end{aligned}
$$

While $T_{N}$ is isomorphic to $\mathbb{C}$, the direct sum of the finite tensor products is not $\ell_{2}(\omega)$, since the isomorphism depends on the ordering. Rather the FocK space is isomorphic with $T=\left\{X \in \ell_{2}(C): X(\varphi)=(-1)^{m} X(\psi)\right.$, whenever $\operatorname{dom} \varphi=\operatorname{dom} \psi=N$ and $|\{i \in N: \varphi(i) \neq \psi(i)\}|=m, N$ and $m$ in $\omega\}$, where $C=\cup\left\{C_{N}: N \in \omega\right\}$.

$M C^{\omega}$ is the countable multiple choice axiom (for each sequence of nonempty sets $S_{n}, n \in \omega$, one may choose a sequence of nonempty finite subsets $\left.E_{n} \subseteq S_{n}, n \in \omega\right)$. In $Z F, M C^{\omega}$ depends on $A C$, but it seems not exclude RusselL's socks [SAGEEv [Sa75] constructs a model of $M C^{\omega}$ where $A C^{\omega}$ fails. His proof appears to extend to not $P D C$. In $Z F A$ the corresponding result is a triviality by section 2.4.] $M C^{\omega}$ implies, that Cantor completeness is equivalent with completeness ([BR83], lemma 3.4.)

Lemma 9. In ZFA+"there are RuSSELL's socks", $T$ (and thus $F$ ) is a locally sequentially compact Hilbert space (norm of $\ell_{2}$ ). There are nonalgebraic bounded quantum like operators on $T$. In $Z F A+M C^{\omega}+$ "there are RUSSELL's socks" each system of linearly independent vectors in $T$ is finite.

Proof. The first and third parts follow as in Brunner [Br90], proof of example 4.4. For $X \in T$ we set $s(X)=\{\varphi \in C: X(\varphi) \neq 0\}$. We let $\prec$ be a lexicographic ordering on $\mathbb{C}$ such that $a \prec 0$ implies $0 \prec-a$. If $X_{n}, n \in \omega$, is a sequence in $T$, then $S=\cup\left\{s\left(X_{n}\right): n \in \omega\right\}$ is finite. For otherwise we define a $P D C$ function $F$ as follows. $N=\left\{n \geq 1: S \cap C_{n} \neq \varnothing\right\}$ would be infinite. If $n \in N$, let $m$ be the least index such that $s\left(X_{m}\right) \cap C_{n} \neq \varnothing$. Then $X_{m}(\varphi) \neq 0$ for all $\varphi \in C_{n}$. For $\psi \in C_{n-1}$ we set $F_{n}(\psi)=\varphi\left(P_{n}\right) \in\left\{a_{n}, b_{n}\right\}$, if $\psi \subseteq \varphi \in C_{n}$ and $X_{m}(\varphi) \succ 0$.

It follows, that $X_{n}$ is a sequence in the finite dimensional space $\ell_{2}(S)$. This proves completeness and locally sequentially compactness.

Since each real diagonal operator $D$ on $\ell_{2}(\omega)$ induces a symmetric bounded operator $A$ on $T$ with the same spectrum, $(A X)(\varphi)=d_{n} X(\varphi)$, if $\varphi \in C_{n}$ and $D e_{n}=d_{n} e_{n}$ for the unit vector $e_{n}$ of $\ell_{2}(\omega)$, the existence of counterexamples to KAPLANSKY's theorem follows from lemma 7.

If $D \subseteq T$ is a system of linearly independent vectors, then in view of the locally sequential compactness of $T[D]^{<\omega}$ is Dedekind finite ([Br86], lemma 2.1). $M C^{\omega}$ implies, that $D$ is finite. [As in lemma 10 below, $S=\left[[D]^{<\omega}\right]^{<\omega}$ is Dedekind finite with an infinite partition $X_{n}=[D]^{n}$ of $[D]^{<\omega}$. If $\varnothing \neq E_{n} \subseteq X_{n}$ is finite, then since $E_{n} \cap E_{m}=\varnothing$ for $n \neq m S$ contains the infinite sequence $\left\langle E_{n}: n \in \omega\right\rangle$. This is improved in lemma 22.] 
e. o. p.

$F$ and also the simpler space $L=\oplus_{n} L_{n}$ are counterexamples to several assertions of Hilbert space theory in $Z F C$.

(i) Both spaces admit no infinite $O N$ system. Thus it is not possible to choose a mode of observation (in the sense of BoHR's complementarity interpretation) by choosing an $O N$ base. Moreover, there is no Hamel base, either. Therefore the multiple choice axiom $M C$ in $Z F A$ does not imply the existence of bases, although it is known to be a consequence thereof ([Ru85], p. 119.) Similar vector spaces have been constructed by LÄUCHLI [La62]. His construction may be modified so as to yield locally sequentially compact Hilbert spaces of the second category such that all closed and everywhere defined linear operators are algebraic (improved in lemma 21).

(ii) The RIEsz representation theorem for continuous linear functionals is invalid, since the duals of $F$ and $L$ differ from $F$ and $L$, whence the notion of a selfadjoint operator does not make sense and the usual approach to quantum theory fails. As the HAHN-BANACH theorem is a consequence of $M C$ (PINCUS [Pi72]), in $Z F A$ RiEsz' theorem does not follow from the HAHN-BANACH theorem. We note, that locally $M C^{\omega}+\exists$ RusseL's socks suffices for such a conclusion. [For then $F$ is Cantor complete and each linear functional attains its norm on the unit sphere. Now an argument due to IshiHara [Is89] proves $H B$ for $F$; c.f. [Mo91].]

If Russell's socks satisfied the EInstein-Bose statistics, then their Fock space $\ell_{2}(\omega)$ would not admit nontrivial quantum like observables. We do not, however, attempt to resolve the physical nature of RUSSELL's socks.

More straightforward examples of nontrivial quantum like observables are obtained from DEDEKIND sets $D$ (infinite, Dedekind finite sets of reals). For $(A x)(d)=d \cdot x(d)$ is a quantum like mapping on $\ell_{2}(D)$ with one dimensional eigenspaces and point spectrum $\sigma_{p}(A)=D$. By contrast, $L$ admits quantum like mappings with countably infinite point spectra and finite multiplicities.

Lemma 10. In $Z F A$, if there is an infinite set $D$ such that its set of finite subsets $[D]^{<\omega}$ is Dedekind finite, then there exist self-adjoint quantum like nonalgebraic bounded observables on $H=\ell_{2}\left([D]^{<\omega}\right)$.

Proof. $\quad H$ is locally sequentially compact (Brunner $[\operatorname{Br} 86]$ ), since $\left[[D]^{<\omega}\right]<\omega$ is Dedekind finite [if $E_{n} \in\left[[D]^{<\omega}\right]^{<\omega}$ is a sequence, we set $\cup E_{n} \in[D]^{<\omega}$ and in view Dedekind finiteness $\cup\left\{\cup E_{n}: n \in \omega\right\}=E \in[D]^{<\omega}$, whence $E_{n} \in \mathcal{P} \mathcal{P}(E)$ is a finite sequence]. However, $\mathcal{P}\left([D]^{<\omega}\right)$ is not Dedekind finite $\left[X_{n}=[D]^{n}, n \in \omega\right]$, whence on $H$ there exist real diagonal operators with infinite point spectra. Lemma 7 concludes the proof.

e. o. p.

Lemma 11. In $Z F A+M C^{\omega}$ any quantum like mapping $A$ on $H=\ell_{2}(D)$ with finite dimensional eigenspaces is algebraic. 
Proof. By lemma 8 and [BR83], lemma 3.4, $H$ is locally sequentially compact, whence by $[\mathrm{Br} 86]$, lemma $2.1,[D]^{<\omega}$ is Dedekind finite and as in lemma $9 D$ is finite.

\section{e. o. p.}

2.4. Weglorz models. As a first step in relating quantum like observables in set theories without $A C$ to quantum theoretical observables in a $Z F C$ universe $V$, we let the finitary observables live in a world which is interpreted within $V$ as a permutation model. In the sequel we shall review the construction of permutation models of $Z F A$.

Inside the real world $V$ which satisfies $Z F C$ we construct for some $X \in V$ a $Z F A+A C$ model $V(X)$, following an idea by J. Truss. In $V(X)$ the set of atoms will correspond to $X$. The definition is by recursion: $V(X)=$ $\cup\left\{V_{\alpha}: \alpha \in O n\right\}$, where $V_{o}=X \times\{0\}$ and $V_{\alpha}=\{(A, \alpha): \alpha$ minimal, such that $\left.A \subseteq \cup\left\{V_{\beta}: \beta \in \alpha\right\}\right\}$. If $x$ and $y$ are objects in $V(X)$, then $x \simeq y$ in $V(X)$, iff $x=y$ in the real world, and $x \widetilde{\in} y$ in $V(X)$, iff $y=(A, \alpha)$ for some $\alpha>0$ and $x \in A$.

The general construction of a FRAENKEL-Mostowski model is as follows. A group $(G, \cdot)$ is given. If $d: G \rightarrow S(X)$ is an injective homomorphism into the symmetric group over $X$, then $d$ is recursively extended to $\widehat{d}$ on all of $V(X): \widehat{d} g(x, 0)=(d g(x), 0)$ and $\widehat{d} g(A, \alpha)=\left(\widehat{d} g^{\prime \prime} A, \alpha\right)$ for $\alpha \geq 0$. If $\underline{G}$ is a $T_{2}$ group topology on $G$ which is generated by a n.h. base at 1 which consists of open subgroups, then an object $x \in V(X)$ is fixed relative to $\underline{G}$, if the stabilizer of $x$, stab $x=\{g \in G: \widehat{d} g(x) \simeq x\}$ is open. $P M$ consists of the hereditarily fixed objects; $P M=\{x \in V(X)$ : all elements in the $\widetilde{\epsilon}$ - transitive closure of $\{x\}$ are fixed relatively to $\underline{G}\}$.

$(P M, \widetilde{\epsilon})$ is a model of $Z F A$. If $A C$ holds in the real world, then $P M$ satisfies $A C$, iff $\underline{G}$ is discrete.

For example, the second FRAENKEL model $F M$ is generated by the group $G=\mathbb{Z}_{2}^{\omega}$ with the product topology and the natural action on $X=$ $\cup\left\{\mathbb{Z}_{2} \times\{n\}: n \in \omega\right\}$. We recall from JECH [Je73], that $F M$ admits RusseLL's socks and thus nontrivial quantum like observables by lemma 9 and $F M$ satisfies the multiple choice axiom $M C$ ([Ru85], p. 74.)

The following speculations serve as a motivation for the next section, where we shall investigate effective descriptions of the quantum theoretical observables. If an element $x$ of the $Z F C$ world $V$ has an isomorphic Dedekind finite copy $y$ in a permutation model $P M$, then $y$ might be considered as an effective representation of $x$. We illustrate this by the following example.

In $V$ we let $\Omega$ be an atomic Boolean algebra with $X$ the set of its atoms. Then $\Omega$ is isomorphic with a set algebra $X_{\Omega} \subseteq \mathcal{P}(A)$ of $V(X), A$ the set of atoms in the sense of sets. We let $\operatorname{Aut}(\Omega)$ be the group of all Boolean algebra automorphisms of $\Omega$. Since $\Omega$ is atomic, the automorphisms are induced by bijections on the set of atoms, whence $\operatorname{Aut}(\Omega)=\operatorname{Aut} X_{\Omega}<S(A)$, the 
symmetric group. Since $X_{\Omega} \supseteq[A]^{<\omega}$, the topology which is generated by the n.h. base at $i d$ of the subgroups $\operatorname{stab}(x), x \in X_{\Omega}$, is $T_{2}$ and it defines a FRAENKEL-Mostowski model $P M_{\Omega}$. In view of its similarity to a construction by WeGLORZ [Wg69] we baptize it WeGLORZ model.

Lemma 12. In $P M_{\Omega}, X_{\Omega}=\mathcal{P}(A)$.

Proof. This result is due to Weglorz [Wg69]. His proof works with $S F(A)$, the group of all finite permutations on $A$. But since $S F(A)<\operatorname{Aut}(\Omega)$, it is valid for $P M_{\Omega}$, too.

e. o. p.

Lemma 13. In $P M_{\Omega} \mathcal{P}(A)$ is Dedekind finite.

Proof. We may assume, that $\Omega$ is infinite. We let $\left\langle E_{n}: n \in \omega\right\rangle$ be a sequence of subsets of $A$ in $P M_{\Omega}$. Then for some sets $x_{1}, \cdots, x_{k} \in X_{\Omega}$ and all $n \operatorname{stab} E_{n} \supseteq \bigcap_{1 \leq i \leq k}$ stab $x_{i}$. If $B$ is the finite Boolean algebra which is generated by the $x_{i}$ and $A$, then we may assume $\left\{x_{1} \cdots, x_{k}\right\}=B$. The atoms of $B$ form a partition of $A$. We show, that $E_{n} \in B$, whence the sequence $\left\langle E_{n}: n \in \omega\right\rangle$ is finite. Pick an atom $y \in B$ and $a \in E_{n} \cap y$. For $b \in y$ we let $\pi \in S F(A)$ be the transposition $\pi=(a ; b)$. Then $\pi$ leaves invariant all the atoms of $B$ (partition) and therefore the other elements of $B$, too, whence $\pi \in \operatorname{stab} E_{n}$ and $b \in E_{n}$. Hence $y \subseteq E_{n}$ and $E_{n}$ is a finite union of the atoms $y \in B$ such that $y \cap E_{n} \neq \varnothing$, whence $E_{n} \in B$.

e. o. p.

As follows from lemma 13, the theory of the atomic Boolean algebras of $V$ has a nontrivial effective content. By contrast, an infinite rigid Boolean algebra does not admit a Dedekind finite representation $B$ in any permutation model $P M$ [In the notation of [Br95] $P M(B)$ satisfies $A C$ by rigidity, whence $B$ would be finite by Dedekind finiteness.] Moreover in all WEgLORz models there are nontrivial quantum like observables, if $\Omega$ is infinite (by lemmas 10 and $13)$.

Since in $P M_{\Omega}$ the set $[A]^{<\omega}$ is Dedekind finite, $P M_{\Omega}$ does not satisfy $M C^{\omega}$, unless $X_{\Omega}$ is finite, whence $F M$ is distinct from these models. Moreover, if $X_{\Omega}$ is infinite, then in $P M_{\Omega}$ there is no choice function on $[A]^{2}$ [for otherwise, if $f$ is a choice function and stab $f \supseteq \bigcap\{\operatorname{stab}(b): b \in B\}, B$ a finite Boolean algebra, then for an infinite atom $y \in B,\{a, b\} \in[y]^{2}$ and $\pi=$ $(a ; b) \quad f\{a, b\}=f \pi\{a, b\}=\pi f\{a, b\}$, contradicting the definition of $\pi]$. Hence the ordered Mostowski model (JECH [Je73], p. 49) is different from $P M_{\Omega}$. In general, $P M_{\Omega}$ is different from the original model of WEGLORz. In WEGLORZ' construction the Boolean algebra $\mathcal{B}_{\Omega}=\mathcal{P}(A) /[A]^{<\omega}$ is wellorderable. In $P M_{\Omega}$ it is infinite, but Dedekind finite, if in $V X_{\Omega}=\mathcal{P}(A)$ is infinite. [If $B \subseteq \mathcal{P}(A)$ is a finite Boolean algebra $(A \in B)$ and $\mathcal{E} \subseteq \mathcal{B}_{\Omega}$ is a finite set such that stab $\mathcal{E} \supseteq \bigcap\{\operatorname{stab} x: x \in B\}$, then for each $E \in \mathcal{E}$ there is a $P \in B$ such that the symmetric difference $E \triangle P$ is finite. For otherwise for some infinite atom (the atoms of $B$ form a finite partition of $A$ ) $x \in B$ and some $E \in \mathcal{E}$ the sets $E \cap x$ and $x \backslash E$ are infinite, whence we may define bijections $\pi_{n}$ such that $\pi_{n} " x=x, \pi_{n}(y)=y$ for $y \in A \backslash x$ and $\pi_{n}(x \cap E) \triangle \pi_{m}(x \cap E)$ is infinite for $n \neq m$ in $\omega$. Then $\pi_{n} \in \operatorname{stab} \mathcal{E}$, but the set of equivalence classes $\left\{\left[\pi_{n} E\right]: n \in \omega\right\} \subseteq \mathcal{E}$ 
is infinite, a contradiction. Hence each sequence of finite subsets of $\mathcal{B}_{\Omega}$ which is supported by $B$ is contained in the finite set $\{[x]: x \in B\}$.

2.5. Benioff extension. The previous lemmata provide us with several examples of quantum like observables in permutation models $P M$. In order to compare their theory with quantum mechanics, we apply an idea due to BeniofF $[\mathrm{Be} 76]$ and investigate the model $P M$ from outside, where $A C$ holds. In $P M \subseteq V(X)$ we let $A$ be a quantum like mapping on the Hilbert space $H$. In $V(X), H$ is an inner product space, whence its completion $\widetilde{H}$ is a Hilbert space. For example, $L \in F M$ (section 2.3) in $V$ is isometrically isomorphic with the space of polynomials $\left.K=\mathbb{C}[x] \cap \mathcal{L}_{2}\right] 0,1\left[\right.$ and $\widetilde{L}$ with $\left.\mathcal{L}_{2}\right] 0,1[$. The quantum like mappings $A$ on $L$ correspond to a proper subset of the family of all linear symmetric mappings on $K$. [The multiplication $(Q f)(x)=x \cdot f(x)$ on $K$ does not correspond to any mapping on $L$. For if in $V Q$ is unitarily equivalent to a linear mapping $A \in P M$ on $H \in P M$, i.e. $A \cdot U=U \cdot Q$ for some bijective isometry $U: H \rightarrow K$ in $V$, then in $P M$ for $h \neq 0$ in $H$ the set $\{p(A)(h): p \in \mathbb{C}[x]\}$ is dense in $H$, since by [Ra73], p. 95-96, $Q$ does not admit a nontrivial closed invariant subspace. Thus in $P M$ the inner product space $H$ is a separable Hausdorff space of the first category. It is therefore wellorderable as a set by [Br83], lemma 2.2, whence in $P M H$ cannot be a Hilbert space.] We show, that there is a unique selfadjoint extension $\widetilde{A}$ of a quantum like $A$ to $\widetilde{H}$; if $A$ is unbounded, then $\operatorname{dom} \widetilde{A} \neq \widetilde{H}$.

Lemma 14. In $Z F C$, if the linear mapping $A$ corresponds to a finitary observable, then its domain $\operatorname{dom} A$ is dense and there exists a unique selfadjoint extension $\widetilde{A}$ of $A$. If conversely $A$ is a selfadjoint operator such that clspan $E V(A)=H$, then $A^{\circ}=A / \operatorname{span} E V(A)$ corresponds to a finitary observable.

Proof. Existence and uniqueness of $\widetilde{A}$ follows from a calculation of the deficiency indices $\operatorname{dim}\left\{x \in \operatorname{dom} A^{*}: A^{*} x= \pm i x\right\}=0$. The adjoint $A^{*}$ is defined from a complete $O N$ system $B$ of eigenvectors of $A$ as the diagonal operator $A^{*} b=A b=\lambda_{b} \cdot b$ for $b \in B$ and $\operatorname{dom} A^{*}=\left\{x \in H: \sum_{b \in B} \lambda_{b}^{2} \cdot|\langle x, b\rangle|^{2}<\infty\right\}$; $A^{*}=\widetilde{A}$ (c.f. VON NeUMANN [vN29]).

e. o. p.

As follows from standard results in spectral theory, in $Z F C A^{\circ}$ is defined for all bounded symmetric operators with a countable spectrum. Another example are the density operators of quantum theory.

Lemma 15. If in the permutation model $P M \subseteq V(X)$ the mapping $A: H \rightarrow H$ on the Hilbert space $H$ is quantum like, then in $V(X)$ there exists a unique self adjoint extension $\widetilde{A}$ of $A$ to the completion $\widetilde{H}$ of $H ; \widetilde{A}^{\circ} \supseteq A$. If $f: \mathbb{R}^{n} \rightarrow \mathbb{R}$ $i s$ an everywhere defined Borel function and in $P M$ the mappings $A_{i}$ correspond to commeasurable quantum like observables on $H$, then in $V(X)$ the self adjoint mappings $\widetilde{A}_{i}$ commute and $f\left(A_{1}, \ldots A_{n}\right)^{\sim}=f\left(\widetilde{A}_{1}, \ldots \widetilde{A}_{n}\right)$.

Proof. It follows from Benioff [Be76], that each $x \in E V(A)$ in $P M$ is an eigenvector of $A$, when viewed in $V(X)$ as an operator on the prae-Hilbert 
space $H$. Hence also in $V(X) H=\operatorname{span} E V(A)$. In $V(X)$ it follows from $A C$, that there is a complete $O N$-system $B \subseteq E V(A) \subseteq H$ of $\widetilde{H}$. The proof of the preceding lemma shows, that in $V(X) A^{*}$ is the unique self adjoint extension $\widetilde{A}$ of $A$. It is obvious, that $\widetilde{A}^{\circ} \supseteq A$. Commeasurability of quantum like observables is equivalent with commutativity by lemma 5 , and the $A_{i}^{*}$ commute, if the finitary $A_{i}$ do. Since then $f\left(\widetilde{A}_{1}, \ldots \widetilde{A}_{n}\right)$ is a self adjoint extension of the quantum like observable $f\left(A_{1}, \ldots A_{n}\right), f\left(A_{1}, \ldots A_{n}\right)^{\sim}=f\left(\widetilde{A}_{1}, \ldots \widetilde{A}_{n}\right)$ follows from uniqueness.

e. o. p.

$\widetilde{A}$ is the Benioff extension of $A$. A self adjoint operator which in the real world $V$ of $Z F C$ is unitarily equivalent to an operator of the form $\widetilde{A}$ for some quantum like mapping $A$ in some permutation model is called intrinsically effective. ( $A$ on $H$ and $B$ on $K$ are unitarily equivalent, if for some bijective isometry $U: H \rightarrow K, U A=B U$ ). If $T$ is a self adjoint operator on the Hilbert space $K$, then $T$ is intrinsically effective, iff dom $T^{\circ}$ is dense in $K$ (c.f. the proof of lemma 16 below), iff $T^{\circ}$ corresponds to a finitary observable on $K$. The correspondence between $T$ and $T^{\circ}$ is delusive, however, since in general in view of the discussion preceding lemma $6 f(T)^{\circ} \neq f\left(T^{\circ}\right)$, whence this correspondence does not respect the functional calculi. On the other hand, as a consequence of lemma 20 below the Benioff extension respects the functional calculus of quantum theory in a strong sense.

Lemma 16. In the real world $V$ of $Z F C$, if $T$ is an intrinsically effective self adjoint operator on the Hilbert space $K$ and $t \in \operatorname{dom} T^{\circ}$, then there is a permutation model PM of $Z F A+M C$ which contains a quantum like mapping $A$ on a locally sequentially compact Hilbert space $L$, such that for some bijective isometry $\widetilde{U}: \widetilde{L} \rightarrow K$ in $V \widetilde{U}^{-1} t \in L$ and in $V T$ is unitarily equivalent with $\widetilde{A}, T \widetilde{U}=\widetilde{U} \widetilde{A}$.

Proof. Since $T$ is intrinsically effective, $T^{\circ}$ contains some finitary mapping on a dense submanifold of $K$, whence span $E V(T)$ is dense in $K$. In $V$ we choose an $O N$-base of $K$ which consists of eigenvectors $k_{\alpha}, \alpha \in \kappa$, of $T ; T k_{\alpha}=\lambda_{\alpha} \cdot k_{\alpha}$. If for some eigenvectors $k_{i}, i \in n$ of $T t=\sum_{i \in n} t_{i} k_{i}$, then we let the $O N-$ base contain these $k_{i}$ and let $H$ be its linear span. In the model $P M$ which is generated by the compact topological group $G=\mathbb{Z}_{2}^{\kappa}$, and thus satisfies $M C$, we repeat the construction of the Hilbert space $L$ which is now related to a transfinite sequence of RUSSELL's socks $P_{\alpha}=\left\{a_{\alpha}, b_{\alpha}\right\}, \alpha \in \kappa ; L=\{x \in$ $\left.\ell_{2}(P): \forall \alpha \in \kappa: x\left(a_{\alpha}\right)+x\left(b_{\alpha}\right)=0\right\}$ where $P=\cup_{\alpha \in \kappa} P_{\alpha}$. On $L$ we define a linear mapping $A \in P M$ with dom $A=L$ through the clause $(A x)(p)=\lambda_{\alpha} \cdot x(p)$, if $p \in P_{\alpha}$. Then in $P M L$ is locally sequentially compact (c.f. lemma 9 ) and in $V$ there is a bijective isometry $U$ between $L$ and $H$ which extends to an isometry $\widetilde{U}$ between $\widetilde{L}$ and $\widetilde{H}=K$; by the definition of $L U^{-1}(t) \in L . P M$ does not depend on $t$, but $U$ does. Since $T / H=U A U^{-1}, T=\widetilde{U} \widetilde{A} \widetilde{U}^{-1}$ and $\widetilde{A}, T$ are unitarily equivalent.

e. o. p.

Locally sequentially compactness is inevitable, for by lemma 8 , if $T$ is the Benioff extension of $A$ in $P M$ which satisfies $M C$ and the multiplicity 
of all eigenvalues of $T$ is finite, then in $P M \operatorname{dom} A$ is locally sequentially compact [modulo an abuse of notation, $\operatorname{ker}(A-\lambda)=\operatorname{dom}(A) \cap \operatorname{ker}(T-\lambda)$ is finite dimensional.] Lemma 16 indicates, that the notion of effectiveness which corresponds to intrinsic effectivity is $Z F A+M C$ (instead of just $Z F A$ ). If in the above proof the powers of $\mathbb{Z}_{2}$ are replaced by $\mathrm{p}$-adic groups, then finite axioms of choice are added (c.f. [Br90].) As follows from the proof of lemma 16, a self adjoint operator $T$ on the Hilbert space $H$ is intrinsically effective, iff its eigenvectors span a dense submanifold of $H$.

Lemma 17. In ZFC, a bounded symmetric operator $T$ on a Hilbert space $H$ is intrinsically effective, iff it is unitarily equivalent to a multiplication operator on a functional Hilbert space.

Proof. This result follows from Halmos' characterization of the latter property (c.f. [Ha82], problem 85), which holds, iff $H=$ clspan $E V(T)$. In view of the above proof this is equivalent with intrinsic effectivity.

\section{e. o. p.}

The notion of intrinsic effectivity may be generalized somewhat. We let $C(H)$ be a definition of a class of symmetric and bounded mappings on a prae-Hilbert space $H$. We assume

(i) absoluteness in the following sense: If in the permutation model $P M \subseteq V(X) A \in C(H), H$ a Hilbert space both in $P M$ and in $V(X)$, then also in $V(X) A \in C(H)$ (c.f. lemma 15);

(ii) finiteness: In $Z F C$, if $H$ is a Hilbert space, then $C(H)=C_{A}(H)$, the algebraic operators (c.f. lemma 6 );

(iii) invariance: In $Z F A$, if $H$ is a Hilbert space, $A \in C(H)$ and the closed subspace $K \subseteq H$ is an invariant subspace for $A$, then $A / K \in$ $C(K)$ (c.f. lemma 4 ).

Since each $A \in C(H)$ is bounded, there is a unique extension $\widetilde{A}$ of $A$ to the completion $\widetilde{H}$ of $H$. In $Z F C$ we thus may define the class $C^{P M}(H)$ for a Hilbert space $H$ and a permutation model $P M$ as follows: $A \in C^{P M}(H)$, iff for some Hilbert space $K$ in the sense of $P M$ up to unitary equivalence $\widetilde{K}=H$, $A / K \in P M$ and in $P M A / K \in C(K)$.

Lemma 18. In $Z F C$, each operator in $C^{P M}(H)$ is intrinsically effective.

Proof. We assume $H=\widetilde{K}, P M \models A \in C(K)$, pick $k \in K$, let $G$ be the stabilizer $\operatorname{stab}(k, K, A, C(K))$ and set $K_{G}=\{x \in K$ : stab $(x) \supseteq G\}$. Since $G$ fixes the topology of $K, K_{G}$ is closed in $P M$. Since in $P M K_{G}$ is wellorderable as a set, $P M$ contains all $V(X)$-sequences of elements in $K_{G}$, whence $K_{G}$ is closed also in $V(X)$ as a subspace of $H$. Since $G$ fixes $A$ and $k \in K_{G}$, $\left\{A^{n} k: n \in \omega\right\} \subseteq K_{G}$ (here $A^{\circ}=i d$ ) and $\operatorname{orb}(k)=\operatorname{cl}_{H} \operatorname{span}\left\{A^{n} k: n \in \omega\right\}$ is closed both in $H$ and in $K$ and so $\operatorname{orb}(k) \subseteq K_{G}$. Moreover, by continuity, $\operatorname{orb}(k)$ is invariant both for $A$ and for $\widetilde{A}$. In view of (iii) in $P M A / \operatorname{orb}(k) \in$ $C(\operatorname{orb}(k))$ and in view of (i) also in $V(X) \widetilde{A} / \operatorname{orb}(k) \in C(\operatorname{orb}(k))$. Hence by (ii) $\widetilde{A} / \operatorname{orb}(k)$ is algebraic, whence $\operatorname{orb}(k)$ is finite dimensional. Therefore in $P M$ by diagonalization $k \in \operatorname{span} E V(A / \operatorname{orb}(k))$ and since $k \in K$ is arbitrary, in $P M$ $A$ is finitary (and quantum like, since $A$ is bounded). 
2.6. Schrödinger equation. Our terminology is motivated by the observation, that for an intrinsically effective Hamiltonian $T$ the dynamics of the corresponding conservative quantum system (i.e. $T$ does not depend on the time) may be evaluated within some permutation model $P M$ of $Z F A$. Initially we let the system under observation be prepared in pure state $\sigma(0) \in \operatorname{dom} T^{\circ}$. The state of the system at time $t, \sigma(t)$, satisfies the Schrödinger equation $T \sigma=i \cdot \partial \sigma / \partial t$. Since the self adjoint observable $T$ is a generator of the unitary group $U(t)=\exp (-i \cdot T \cdot t)$ in the Hilbert space $K$ of the equation, its solution is $\sigma(t)=U(t)(\sigma(0))$. As $T$ is intrinsically effective, up to a unitary equivalence $U: \widetilde{H} \rightarrow K$ in $V T=U \widetilde{A} U^{-1}$ for some quantum like $A: H \rightarrow H$ in some permutation model $P M$ such that $U^{-1} \sigma(0) \in H$ (lemma 16). In $P M$ we set $V(t) x=\exp (-i \cdot \lambda \cdot t) \cdot x$ for $x \in E V(A)$ with $A x=\lambda x$ and extend $V(t)$ linearly over $H$. Then $V(t) \in P M$ is equivalent to the restriction (in fact, a compression) of the unitary group $U(t)$ to $H$ (lemma 15) and the solutions of the Schrödinger equation may be evaluated within $P M$ as $U^{-1} \sigma(t)=U^{-1} U(t)(\sigma(0))=V(t)\left(U^{-1} \sigma(0)\right) \in P M$.

If moreover all eigenspaces of $T$ are finite dimensional, then independently of $P M$ in $V$ for each bijective isometry $U: \widetilde{H} \rightarrow K H=U^{-1}$ " dom $T^{\circ}$. In $V$, if $D$ is any state, then the expected evolution of a commeasurable quantum system $S=g(T)$ at this state may be computed within $P M$, too. (In view of lemma 20, if $K$ is separable and $S$ is intrinsically effective, then $S T=T S$ suffices.) For there is an operator $B \in P M$ such that for all Borel functions $f$ the expected value of $f(T)$ at $D, \operatorname{tr}(f(T) D)$, is equal to the expected value in $P M$ of $f(A)$ at $B, \operatorname{tr}(f(A) B)$, where $\operatorname{tr}(C)=\sup \left\{\sum_{x \in E}\langle C x, x\rangle: E\right.$ a finite $O N-$ system in dom $C\}$. [In $P M$, for $\lambda \in \sigma_{p}(T)$ we let $P_{\lambda}$ be the orthogonal projection onto the Cantor complete subspace $\operatorname{ker}(A-\lambda)$ and $n_{\lambda}$ its dimension. We set $B=\sum_{\lambda \in \sigma_{p}(T)} \frac{1}{n_{\lambda}} \cdot \operatorname{tr}\left(U \widetilde{P}_{\lambda} U^{-1} D\right) \cdot P_{\lambda} \in P M ; \operatorname{tr}\left(P_{\lambda} B\right)=\operatorname{tr}\left(U \widetilde{P}_{\lambda} U^{-1} D\right)$. The extension $U \widetilde{P}_{\lambda} U^{-1}$ of $P_{\lambda}$ is the projection onto $\operatorname{ker}(T-\lambda)$. Then $\operatorname{tr}(f(T) D)=$ $\left.\sum_{\lambda \in \sigma_{p}(T)} f(\lambda) \operatorname{tr}\left(U \widetilde{P}_{\lambda} U^{-1} D\right)=\sum_{\lambda \in \sigma_{p}(T)} f(\lambda) \operatorname{tr}\left(P_{\lambda} B\right)=\operatorname{tr}(f(A) B).\right]$

For example, the Hamiltonian $T=\frac{\hbar}{2 m} \cdot P^{2}+\frac{f}{2 \hbar} \cdot Q^{2}$ on $\mathcal{L}_{2}(\mathbb{R})$, where $(P g)(x)=-i \cdot \frac{d g}{d x}(x)$ and $(Q g)(x)=x \cdot g(x)$ are the momentum and position operators, $\hbar=1.05 \cdot 10^{-27} \mathrm{erg}$ sec, is an unbounded intrinsically effective observable with one - dimensional eigenspaces (JAUCH [Ja68], pp. 211 - 219, computes an ON-base of eigenvectors). According to the Ehrenfest rule it corresponds to the harmonic oscillator of classical mechanics with kinetic energy $\frac{1}{2 m} \cdot p^{2}$ and potential $\frac{f}{2} q^{2}$. More generally, by a theorem due to H. WEYL, if in classical mechanics the potential $v(q)$ is continuous and $\lim v(q)=\infty$ as $q \rightarrow \pm \infty$, then the Hamiltonian of the corresponding elementary particle is intrinsically effective (c.f. TitchmARsh [Ti62], pp. $110-113,121-122$ and $127)$ and all its eigenspaces are finite dimensional ([Du63], p. 1285). Similar results are true for three dimensional elementary particles.

The dependence of the representation of the state $D$ by an operator $B$ in a permutation model $P M$ of $M C$ on the Hamiltonian $T$ is unavoidable even 
if $D$ is a mixture of states in $\operatorname{dom} T^{\circ}$. In $\ell_{2}(\mathbb{Z})$ we let $T$ be an intrinsically effective Hamiltonian with eigenspaces $\operatorname{span}\left\{e_{k}\right\}, k \in \mathbb{Z}$. If $T=U \widetilde{A} U^{-1}$ for some quantum like mapping $A$ on the Hilbert space $H$ of some model $P M$ of $Z F A+M C$ and some bijective isometry $U: \widetilde{H} \rightarrow \ell_{2}(\mathbb{Z})$, then in $V H=U^{-1} " \operatorname{dom} T^{\circ}=U^{-1} " \operatorname{span}\left\{e_{k}: k \in \mathbb{Z}\right\}$ and in $P M H$ is locally sequentially compact (lemma 8 and $M C$.) For a sequence $w_{k}>0$ of weights, $\sum_{k \in \mathbb{Z}} w_{k}=1$, we set $D=\sum_{k \in \mathbb{Z}} w_{k} P_{k}, P_{k}$ the orthogonal projection onto span $\left\{e_{k}+e_{k+1}\right\}$. Then in $P M$ there is no bounded symmetric operator $B$ on $H$ such that $\operatorname{tr}\left(U \widetilde{P} U^{-1} D\right)=\operatorname{tr}(P B)$ for all orthogonal projections $P$ of $P M$ in $H$, rather than projections onto eigenspaces of $T^{\circ}$ only. [For otherwise, when applied to the projection $P$ onto span $\{x\}, x \in H$ a unit vector, $\langle D U x, U x\rangle=$ $\langle B x, x\rangle$. The polarization identity implies $\langle D U x, U y\rangle=\langle B x, y\rangle$ for all $x, y \in H$, whence $B$ is the restriction of $U^{-1} D U$ to $H$. Since $H$ is locally sequentially compact, $H=\operatorname{span} E V(B)=\operatorname{span}\left(H \cap E V\left(U^{-1} D U\right)\right)$. This contradicts $E V(D) \cap \operatorname{dom} T^{\circ}=\varnothing$.] Thus in the notation of quantum theory lemma 8 proves the impossibility of a certain type of hidden parameters.

As is easily seen from the proof of lemma 18 the position operator $(Q f)(x)=x \cdot f(x)$ on $\left.\mathcal{L}_{2}\right] 0,1[$ is far from being intrinsically effective. [Since by [Ra73], pp. $65-66$, the only finite dimensional invariant subspace of $Q$ is $\{0\}, k \in \operatorname{orb}(k)=\{0\}$ for all $k \in K$.] Hence the classical HeisenBerG uncertainty relation for the position and the momentum operators does not apply in an effective quantum theory. There is, however, a different phenomenon of complementarity.

Lemma 19. In $Z F A$, there exist unitarily equivalent bounded self adjoint operators $S, T$ on $\ell_{2}(\omega)$ such that span $E V(S)$ and span $E V(T)$ are dense, but $V\left(S^{o}, w\right) \cdot V\left(T^{o}, w\right) \geq \frac{1}{4}$ for all unit vectors $w \in \operatorname{dom} S^{o} \cap \operatorname{dom} T^{o}$.

Proof. As is wellknown, if one of the self-adjoint observables $S, T$ is bounded, then the product of the variances $V(S, w) \cdot V(T, w)$, where

$$
V(S, w)=\langle S w, S w\rangle-\langle w, S w\rangle^{2}
$$

can be made arbitrarily small at some pure state $w$ (i.e. $\|w\|=1$ ).

We let $e_{i}, i \geq 0$, be the standard $O N$ base for $\ell_{2}(\omega)$ and $f_{i}, i \geq 1$, be an $O N$ base for $e_{o}^{\perp}$ which satisfies span $\left\{e_{i}: i \geq 1\right\} \cap \operatorname{span}\left\{f_{i}: i \geq 1\right\}=\{0\} ; f_{o}=e_{o}$. From $e_{i}$ we define the $O N$ base $w_{o}=\frac{e_{o}+e_{1}}{\sqrt{2}}, w_{1}=\frac{e_{o}-e_{1}}{\sqrt{2}}, w_{i}=e_{i}$ for $i \geq 2$ and similarily we define $\bar{w}_{i}$ from $f_{i}$. If $S$ is the diagonal operator $S w_{i}=\alpha_{i} w_{i}$, all $\alpha_{i}$ real and distinct, and $T \bar{w}_{i}=\alpha_{i} \bar{w}_{i}$, then $\operatorname{dom} S^{o} \cap \operatorname{dom} T^{o}=\operatorname{span}\left\{e_{o}\right\}$ and $V\left(S, e_{o}\right)=V\left(T, e_{o}\right)=\frac{\left(\alpha_{o}-\alpha_{1}\right)^{2}}{2}$.

\section{e. o. p.}

Complementarity may be avoided, if the intrinsically effective Hamiltonians $S$ and $T$ on $K$ are compatible: There exist a locally sequentially compact Hilbert space $H$ in some permutation model $P M$ (c.f. lemma 16), a surjective isometry $U: \underset{\widetilde{H}}{\widetilde{A}} \rightarrow K$ and quantum like mappings $A, B$ on $H$ in $P M$ such that $S U=U \widetilde{A}$ and $T U=U \widetilde{B}$. [If $w$ is an eigenvector of $A$ or $B$, then $V(S, U w) \cdot V(T, U w)=0$ and $U w \in \operatorname{dom} S \cap \operatorname{dom} T$.] 
We recall from spectral theory, that a concatenation of experiments $S, T$ is again an experiment; i.e. the concatenation corresponds to a Hamiltonian $R$ on $K$ such that (disregarding the durations of the experiments which we set to be one unit of time) $\exp (-i \cdot S) \cdot \exp (-i \cdot T)=\exp (-i \cdot R)$. In view of the nonuniqueness of $R$, an exact specification of the concatenation of noncommeasurable experiments depends on additional restrictions on the empirical context, such as compatibility. As an application to control theory we note, that if $S, T$ are intrinsically effective and compatible, then $R$ may be chosen to be intrinsically effective, too, and compatible with both $S$ and $T$; i.e. $R U=U \widetilde{C}$ on $\widetilde{H}$ for some bounded quantum like $C \in P M$ on $H$ such that $\exp (-i \cdot A) \cdot \exp (-i \cdot B)=\exp (-i \cdot C)$. [Since in $P M H$ is locally sequentially compact, for finite $F \subseteq H$ the submanifold $E=$ span $\{p(A, B)(h): p \in \mathbb{C}[x, y], h \in F\}$ is finite dimensional and invariant for both $A$ and $B$. In $E$ we set $\mathcal{M}=\{-i \cdot M: M$ a Hermitean matrix $\}=\{N: N$ skew Hermitean $\}$. By the spectral theorem for normal matrices the exponential of this Lie algebra is the group $\exp ^{\prime \prime} \mathcal{M}=\mathcal{U}$ of the unitary matrices, whence for the restrictions $R=\exp (-i \cdot A / E) \cdot \exp (-i \cdot B / E)=\exp \left(-i \cdot C_{F}\right) \in \mathcal{U}$ for some uniquely determined Hermitean $C_{F}=f(R)$, where $f\{z:|z|=1\} \subseteq[0,2 \pi[$. Then by the proof of lemma 3 in $P M C=\cup\left\{C_{F}: F \subseteq H\right.$ finite $\}$ is a symmetric finitary and everywhere defined bounded mapping on $H$ which satisfies the desired exponential equation.]

As follows from lemma 16, if $T$ and $f(T)$ are self adjoint operators, such that $T$ is intrinsically effective, then both are extensions of commeasurable quantum like mappings $A$ and $f(A)$ on some Hilbert space in some model $P M$. The hypothesis in this observation my be weakened, so that intrinsically effective observables which are commeasurable in the sense of quantum theory may be observed simultaneously by means of the measurement of another intrinsically effective observable and thus are compatible.

Lemma 20. In the real world $V$ of $Z F C$, if $S$ and $T$ are intrinsically effective self adjoint commuting operators on the separable Hilbert space $K$, then there is a permutation model PM of ZFA+MC which contains a bounded quantum like mapping $A$ on a locally sequentially compact Hilbert space $H$, such that in $V S$ and $T$ are unitarily equivalent (by means of the same isometry) with functions of $\widetilde{A}$.

Proof. We first observe, that intrinsic effectivity is hereditary. If $T$ is intrinsically effective and $L$ is a closed invariant subspace, then the restriction $T / L$ is intrinsically effective on $L$. In view of lemma 16 it suffices to observe, that dom $(T / L)^{\circ}$ is dense in $L$. For if $P$ is the orthogonal projection onto $L$, $x \in L$ and $x \perp \operatorname{dom}(T / L)^{\circ}$, then $\langle x, P y\rangle=0$ for $y \in E V(T)$ [c.f. the remarks following lemma 4], whence $\langle x, y\rangle=\langle x, P y\rangle+\langle x, y-P y\rangle=0$, since $(y-P y) \perp L$; therefore $x \perp \operatorname{dom} T^{\circ}$ and $x=0$.

If $S$ and $T$ are commuting intrinsically effective operators, then for $\lambda \in \sigma_{p}(T)$ the closed manifold $L_{\lambda}=\operatorname{ker}(T-\lambda)$ is a nontrivial invariant subspace for $S$ and we may choose a complete $O N$-base $B_{\lambda} \subseteq E V(S / L)$ for $L_{\lambda} \subseteq E V(T)$. Since $T$ is intrinsically effective, $B=\cup\left\{B_{\lambda}: \lambda \in \sigma_{p}(T)\right\} \subseteq E V(S) \cap E V(T)$ is a complete $O N$-base for $K$ and since $K$ is separable, it is countable. We let $\beta: B \rightarrow \mathbb{R}$ be a bounded injective mapping and define up to unitary equivalence 
a bounded quantum like mapping $A$ on $H=\operatorname{span} B$ in some model $P M$ as in lemma 16 through $A b=\beta(b) \cdot b$. Then $f(\beta(b)) \cdot b=S b$ and $g(\beta(b)) \cdot b=T b$ define real mappings $f, g$ such that $S, T$ are Benioff extensions of $f(A)$ and $g(A)$.

e. o. p.

2.7. Quantum logic. The FraEnKEL-HALPERn model $F H=P M_{\Omega}$, where $X_{\Omega}$ is the algebra of all finite and cofinite subsets of $A$, is known to be the least permutation model. FH admits amorphous sets (infinite sets whose infinite subsets are cofinite), for example, the set $A$ of the atoms is amorphous, and it satisfies the partial finite choice axiom $P A C_{\text {fin }}$ (each infinite family of finite sets admits an infinite subfamily with a choice function.) $F H$ is a source of several counterexamples.

In FH the projection lattice (i.e. the family of the closed subspaces of $\ell_{2}(A)$ which admit orthogonal projections; we do not know if in general without $A C$ it is a lattice) is modular (i.e. it satisfies the shearing identity $x \wedge(y \vee z)=x \wedge((y \wedge(x \vee z)) \vee z))$. BIRKhoff and Von NEUMANN [Bi36] have considered modularity as a requirement for quantum logic, but in $Z F C$ it is satisfied only for systems with a finite degree of freedom. [For a proof of modularity it suffices to note, that $S_{1}+S_{2}$ is closed, if the $S_{i}$ are the ranges of the orthogonal projections $P_{i}$. By [Br86], corollary 5.2, when applied to $P_{i}$, the subspace $S_{i}$ is a direct sum of a finite dimensional subspace and some $\ell_{2}\left(F_{i}\right)$, $F_{i}$ cofinite or empty. Obviously these subspaces form a lattice. $S_{1}+S_{2}$, as a sum of finite dimensional spaces and the closed subspace $\ell_{2}\left(F_{1} \cup F_{2}\right)$, is closed, whence $S_{1}+S_{2}=S_{1} \vee S_{2}$ is the range of the orthogonal projection $P_{1} \vee P_{2}$. Hence for $P_{2} \leq Q$ the following identity is proved as in [Ha82], solution 15 on $\mathrm{p}$. 177: $\left.\left(P_{1} \vee P_{2}\right) \wedge Q=\left(P_{1} \wedge Q\right) \vee P_{2} \cdot\right]$

In $F H$, the Hilbert space $\ell_{2}(A)$ is a counterexample to the GLEASON and MAEDA [Ma80] theorem about the representation of the transfinitely additive states by density matrices. For if $P$ is a projection, then we set $\alpha(P)=0$, if the range of $P$ is finite dimensional, and $\alpha(P)=1$, otherwise. In $F H$ for each transfinite sequence $\left\langle P_{\lambda}: \lambda \in \kappa\right\rangle$ of pairwise orthogonal projections $(P \cdot Q=0)$ $\alpha\left(\bigvee_{\lambda \in \kappa} P_{\lambda}\right)=\sum_{\lambda \in \kappa} \alpha\left(P_{\lambda}\right)=\sup \left\{\sum_{\lambda \in K} \alpha\left(P_{\lambda}\right): K \in[\kappa]^{<\omega}\right\}$ converges, whence $\alpha$ is a transfinitely additive state. [We let $e$ be the least support of the sequence $\left\langle P_{\lambda}: \lambda \in \kappa\right\rangle$. In view of [Br86], corollary $5.2, P_{\lambda}$ is a direct sum of a projection in $\ell_{2}(e)$ and a scalar $\rho_{\lambda} \in\{0,1\}$ on $\ell_{2}(A \backslash e)$. Hence either all $\rho_{\lambda}=0$ and $\alpha\left(\bigvee_{\lambda} P_{\lambda}\right)=\sum_{\lambda} \alpha\left(P_{\lambda}\right)=0$, or in view of orthogonality exactly one $\rho_{\lambda}=1$ and $\alpha\left(\bigvee_{\lambda} P_{\lambda}\right)=\sum_{\lambda} \alpha\left(P_{\lambda}\right)=1$. In both cases all except finitely many projections vanish.] There is, however, no bounded operator $D$ on $\ell_{2}(A)$ such that for all orthogonal projections $P$ the expected value is $\alpha(P)=\operatorname{tr}(P D)$ [If $e$ is the least support of $D$ in $F H$, then $D$ is a direct sum of a finite matrix on $\ell_{2}(e)$ and a scalar $\rho$ on $E=\ell_{2}(A \backslash e)$. We let $P_{E}$ be the orthogonal projection onto $E$, $P_{E} x=x \mid E$. Then $\alpha\left(P_{E}\right)=1$, but $\operatorname{tr}\left(P_{E} D\right)=\infty$, if $\rho \neq 0$, or $\operatorname{tr}\left(P_{E} D\right)=0$, if $\rho=0$.] A similar construction shows, that the ultrafilter extension theorem $B P I$ does not imply the GLEASON-MAEDA theorem for locally sequentially compact Hilbert spaces, since it fails in the ordered Mostowski-model. [In $\ell_{2}(A), A$ 
the set of atoms of that model, consider $\alpha(P)=1$, if $\left\{a \in A: P e_{a}=e_{a}\right\}$ is unbounded from above, and $\alpha(P)=0$, otherwise.]

The hidden parameter issue involves only the finite dimensional effective versions of this theorem which, however, are not constructive. The GLEASONMAEDA theorem for completely additive states (additivity for possibly nonwellorderable families of closed and pairwise orthogonal subspaces) is effective, too.

In $F H$ there is a counterexample to a converse of lemma 6 , namely the assertion "if all quantum like observables on $H$ are algebraic, then there is an orthonormal base of $H$ " which therefore depends on $A C$.

Lemma 21. In FH there is a locally sequentially compact Hilbert space $H$ such that each linear map $T: H \rightarrow H$, dom $T=H$, is a direct sum of a finite matrix and a scalar (with an infinite dimensional eigenspace).

Proof. We set $H=\left\{x \in \ell_{2}(A): \sum_{a \in A} x(a)=0\right\}$ where $A$ are the atoms of $F H$. Since by [Br86], lemmas 2.1 and 2.2 separable subspaces of $\ell_{2}(A)$ are finite dimensional ( $A$ is amorphous and thus $[A]^{<\omega}$ is Dedekind finite; c.f. JECH [Je73]), $H$ is locally sequentially compact and complete. We let $e_{a}, a \in A$, be the canonical unit vectors which form an $O N$-base of $\ell_{2}(A)$.

We first observe, that linear functionals on $H$ extend to bounded linear functionals on $\ell_{2}(A)$. In $F H$ there are least supports ([Je73]). If $f: H \rightarrow \mathbb{C}$ is any linear functional and $e=\operatorname{supp}(f)$ is its least support, then $f\left(e_{a}-e_{b}\right)=$ $f\left(e_{a}-e_{c}\right)$ for $a \in e, b, c \in A \backslash e$. We set $g_{a}=f\left(e_{a}-e_{b}\right)$ and $g(x)=$ $\sum_{a \in e} g_{a} \cdot\left\langle x, e_{a}\right\rangle\left(g: \ell_{2}(D) \rightarrow \mathbb{C}\right)$. If $x \in H$, then $f(x)=g(x)$, for if $x=e_{b}-e_{c}$, $b, c \in A \backslash e$, then $f(x)=f\left(e_{c}-e_{b}\right)$, whence by linearity $f(x)=0=g(x)$. If $x=e_{a}-e_{b}, a \in e$ and $b \notin e$, then by the definition of $g \quad f(x)=g(x)$. If $x=e_{a}-e_{b}, a \in e$ and $b \in e$, then for some $c \notin e \quad f(x)=f\left(e_{a}-e_{c}\right)-f\left(e_{b}-e_{c}\right)=$ $g\left(e_{a}-e_{c}\right)-g\left(e_{b}-e_{c}\right)=g(x)$ by linearity. Since the vectors $e_{a}-e_{b} \operatorname{span} H$, $f=g / H$.

If $T: H \rightarrow H$ is linear and $e=\operatorname{supp}(T)$, then $f_{a}: H \rightarrow \mathbb{C}, f_{a}(x)=$ $\left\langle T x, e_{a}\right\rangle$ is a linear mapping with $\operatorname{supp}\left(f_{a}\right) \subseteq e \cup\{a\}$. Hence the numbers $t(b, a)=\left\langle T\left(e_{b}-e_{c}\right), e_{a}\right\rangle=f_{a}\left(e_{b}-e_{c}\right)$ do not depend on $c \in A \backslash(e \cup\{a\})$. If we set $\lambda(a)=t(a, a)$ for $a \in A \backslash e$ and $\lambda(a)=0$, otherwise, then for $x \in H T$ satisfies $\left\langle T x, e_{a}\right\rangle=f_{a}(x)=\sum_{b \in e} t(b, a) \cdot\left\langle x, e_{b}\right\rangle+\lambda(a) \cdot\left\langle x, e_{a}\right\rangle$, as follows from the above representation of the linear functionals. Since the function $t: e \times A \rightarrow \mathbb{C}$ has support $\operatorname{supp}(t) \subseteq e, t(b, a)=0$ for $a \notin e$. For $t(b, a)=\left\langle T\left(e_{b}-e_{c}\right), e_{a}\right\rangle$ for some $c \notin e, c \neq a$, and $y=T\left(e_{b}-e_{c}\right) \in \ell_{2}(A)$ satisfies $y(d)=0$, if $d \notin \operatorname{supp}(y) \subseteq \operatorname{supp} T \cup\{b, c\} \subseteq e \cup\{c\}$; i.e. $y(a)=\left\langle y, e_{a}\right\rangle=0$. It follows, that $T x=\sum_{a \in e} \sum_{b \in e} t(b, a)\left\langle x, e_{b}\right\rangle \cdot e_{a}+\sum_{a \in A} \lambda(a) \cdot\left\langle x, e_{a}\right\rangle \cdot e_{a}$. Since $\lambda(a)=\lambda$ for some $\lambda \in \mathbb{C}$ and all $a \in A \backslash e, T$ is the direct sum of the restriction to $H$ of a finite matrix $F$ on $\ell_{2}(e), F(a, b)=t(a, b)$ for $a, b$ in $e$, and the scalar $\lambda$ on $H \cap \ell_{2}(A \backslash e)$. As an additional requirement on $F$ we note, that all column sums are equal to $\lambda\left(\sum_{a \in A}\left\langle T\left(e_{b}-e_{c}\right), e_{a}\right\rangle=\sum_{a \in e}\left\langle F e_{b}, e_{a}\right\rangle-\lambda=0\right.$, since $T\left(e_{b}-e_{c}\right) \in H$, whenever $\left.b \in e, \quad c \in A \backslash e\right)$.

There does not exist an $O N$-base $B$ for $H$, for otherwise $H$ is isomorphic to $\ell_{2}(B),[B]^{<\omega}$ Dedekind finite, and $f(x)=\sum_{b \in B} x(b)$ is an everywhere 
defined unbounded linear functional on $\ell_{2}(B)$ and thus on $H$.

e. o. p.

Although $H$ is sequentially closed as a submanifold of $\ell_{2}(A)$, it is dense in $\ell_{2}(A)$. [For given $x \in \ell_{2}(A), \varepsilon>0$ and $E \subseteq A$ finite, such that $\sum_{a \in A \backslash E}|x(a)|^{2}<\varepsilon / 2$, then we set $y=\sum_{a \in E} x(a)$, choose a set $F \subseteq A \backslash E$ with $n>\frac{2 \cdot|y|}{\varepsilon}$ elements and define $z \in H$ as $z(a)=x(a)$, if $a \in E ; z(a)=-y / n$, if $a \in F ; z(a)=0$, otherwise. Then $\left.\|x-z\|_{2}<\varepsilon\right]$. In view of lemma 21, the projection lattice of $H$ is modular and there are counterexamples to GLEASON's theorem. The following construction generalizes lemma 21.

Lemma 22. In ZFA, if each locally sequentially compact Hilbert space is Cantor complete, and $[D]^{<\omega}$ is Dedekind finite, then $D$ is finite.

Proof. Since $[D]^{<\omega}$ is Dedekind finite, we may define $H=\left\{x \in \ell_{2}(D)\right.$ : $\left.\sum_{a \in D} x(a)=0\right\}$ and $K=H \oplus \ell_{2}(D)$. Since $K$ is locally sequentially compact, it is Cantor complete in view of our hypothesis. The linear subspace $S=\{(x, x)$ : $x \in H\}$ is closed, since it is the graph of the embedding of $H$ into $\ell_{2}(D)$, but it does not admit an orthogonal projection $P$ of $K$ onto $S$. For if $k=(x, y) \in K$ and $P k=(z, z) \in S, x, z \in H, y \in \ell_{2}(D)$, then for all $s=(u, u) \in S, u \in H$, it holds, that $k-P k \perp s$, whence in $\ell_{2}(D)$ the scalar product $\langle x+y-2 \cdot z, u\rangle=0$ for all $u \in H$. Hence $x+y+2 \cdot z=0$, which is impossible for $y \notin H$. [For if $w \neq 0$, say $w(b) \neq 0$, where $w=x+y+2 \cdot z$, then $\langle w, u\rangle=w(b) \neq 0$ for the following element $u \in H: u(b)=1 ; u(c)=-1$ for some $c$ such that $w(c)=0$; $u(a)=0$, otherwise.] From the arguments following lemma 4 we conclude, that $K$ is not Cantor complete, since otherwise each closed linear subspace of $K$ would be the range of an orthogonal projection in $K$.

e. o. p.

2.8. Maximal completion. If $H$ is the Hilbert space of lemma 21, then $K=F H \cap \widetilde{H}$ is another Hilbert space of $F H$ such that $K \neq H$. [H is dense in $\left.\ell_{2}(A)\right]$. If on the other hand $H$ is defined in some model $P M$ via lemma 10, then $\widetilde{H} \cap P M=H$. The same is true for the Hilbert space of RusseLL's socks. (We note, that these results depend on the particular canonical embedding $e: H \rightarrow \widetilde{H}$ which is constructed below. Without mentioning $e$ it might be read as an abbreviation for: "If in $P M H$ is dense in the Hilbert space $K$, then $\left.H=K^{\prime \prime}\right)$.

Lemma 23. If in the permutation model $P M \subseteq V(X) H$ is a Hilbert space, and if $\widetilde{H}$ is its completion in $V(X)$, then $K=\widetilde{H} \cap P M \in P M$ and as a submanifold of $\widetilde{H}$ in $P M K$ is a Hilbert space. If $P M$ satisfies $M C^{\omega}$, then $K=H$.

Proof. We need to consider a concrete construction of the completion. To this end in $V(X)$ we let $e: H \rightarrow \ell_{\infty}\left(S_{1}\right), S_{1}=\{s \in H:\|s\|=1\}$, be the embedding $e(h)=\varphi_{h}$, where $\varphi_{h}(s)=<h, s>$. [Since $\left|\varphi_{h}(s)\right| \leq\|h\|,\left\|\varphi_{h}\right\|_{\infty}<\infty$.] Then $e$ is easily seen to be a linear isometry $\left[\left|\varphi_{h}(s)\right|=\|h\|\right.$ for $s=h /\|h\|$ and $\left.h \neq 0\right]$. Moreover, since $S_{1} \in P M$, each function $\varphi \in \ell_{\infty}\left(S_{1}\right)$ is a subset of $P M$. 
We observe, that $\mathcal{L}=\ell_{\infty}\left(S_{1}\right) \cap P M \in P M$. For if $P M$ is generated by the topological group $G$ and $g \in \operatorname{stab}(H)$, then for $\varphi \in \ell_{\infty}\left(S_{1}\right)$

$$
\psi=(\widehat{d} g)(\varphi)=\left\{<(\widehat{d} g)(s), \varphi(s)>: s \in S_{1}\right\} \in V(X)
$$

is a complex valued function with domain $S_{1}$ [since $(\widehat{d g})^{\prime \prime} S_{1}=S_{1}$ ] and $\|\psi\|_{\infty}=$ $\|\varphi\|_{\infty}$. Hence $(\widehat{d} g)^{\prime \prime} \ell_{\infty}\left(S_{1}\right) \subseteq \ell_{\infty}\left(S_{1}\right)$ and $\operatorname{stab}(\mathcal{L}) \supseteq \operatorname{stab}(H)$ is open. $\mathcal{L}$ is $\ell_{\infty}\left(S_{1}\right)$ in the sense of $P M$ and the vector space operations and norm restrict from $V(X)$ to the equally defined functions of $P M$. Moreover, in $P M \mathcal{L}$ is complete [completeness of $\ell_{\infty}\left(S_{1}\right)$ is provable in $Z F A$.] in $V(X)$

$\widetilde{H}$ is the closure of $e^{\prime \prime} H$ in the sense of $V(X)$. Since $K=\widetilde{H} \cap P M \subseteq \mathcal{L}$,

$$
K=\left\{x \in \mathcal{L}: \forall \varepsilon>0 \exists h \in H:\|x-e(h)\|_{\infty}<\varepsilon\right\}
$$

Similarily, in $P M$ we use this definition to define $K_{1} \in P M$. Since all parameters in this definition are in $P M$ and have the same meaning in $P M$ as in $V(X)$, we conclude, that $K=K_{1} \in P M$ is the closure of $e^{\prime \prime} H$ in $P M$.

The norm and the vector space operations of $K$ are inherited from $\ell_{\infty}\left(S_{1}\right)$ and thus from $\mathcal{L}$, whence in $P M K$ is a prae-Hilbert space with these functions. Since in $P M K$ is closed in $\mathcal{L}, K$ is complete.

Now let $P M$ satisfy $M C^{\omega}$. In the sequel we shall work inside $P M$. We pick $x \in K=\widetilde{H} \cap P M \in P M$ and note, that since $e^{\prime \prime} H$ is dense in $K, W_{n}=\left\{h \in H:\|x-e(h)\|_{\infty}<\frac{1}{n}\right\}$ is nonempty for $n \geq 1$. By $M C^{\omega}$ there is a sequence $\varnothing \neq F_{n} \subseteq W_{n}$ of finite sets. We let $y_{n}$ be the average of $F_{n}$. Then $y_{n} \in W_{n}$, whence $\lim e\left(y_{n}\right)=x$. Since $H$ is sequentially complete and the sequence $\left\langle y_{n}: n \in \omega>\right.$ is Cauchy [because the sequence $<e\left(y_{n}\right): n \in \omega>$ is Cauchy], there is some $y \in H$ such that $\lim y_{n}=y$, whence $x=\lim e\left(y_{n}\right)=e(y) \in e^{\prime \prime} H$; i.e. $K=e^{\prime \prime} H$.

\section{e. o. p.}

Usually the representative of the isometry class of the completion of $H$, $\widetilde{H}$, will be wellorderable as a set even in $P M$. Then the embedding $e: H \rightarrow \widetilde{H}$ cannot be traced in $P M$ unless $H$ is wellorderable and $H=\widetilde{H}$. It is, however, approximated by the relations $E_{G} \in P M ; x E_{G} y$, if $x \in H, y \in \widetilde{H} \in V(\varnothing)$ and $y=e(\widehat{d} \pi x)$ for some $\pi \in G, G$ an open subgroup of stab $H . E_{G}$ induces a partition $\mathcal{P}_{G}$ on $\widetilde{H} ; \mathcal{P}_{G}=\left\{\widetilde{H} \backslash e^{"} H,\{e(\widehat{d} \pi x): \pi \in G\}: x \in H\right\}$. For example, if in FMH is the Hilbert space $L$ of RussELL's socks, $G=$ stab $\varnothing$ and $\widetilde{H}=\ell_{2}$, then $x E_{G} y$, iff $x(a) \in\left\{y_{n},-y_{n}\right\}$ for $a \in P_{n}, e^{\prime \prime} H=\{y \in$ $\left.\ell_{2}:\left\{n \in \omega: y_{n} \neq 0\right\} \in[\omega]^{<\omega}\right\}$ and the $\mathcal{P}_{G}$ equivalence class of $y \in e^{\prime \prime} H$ is $\left\{\left\langle\varepsilon_{n} y_{n}: n \in \omega\right\rangle: \varepsilon \in\{+1,-1\}^{\omega}\right\}$. If it is assumed, that a pure state in $\widetilde{H}$ provides a complete and exhaustive description of the system in question, then $\mathcal{P}_{G}$ reflects the state of knowledge about this system due to the information $G$ (e.g. the orderings on finitely many pairs of socks). The following technical question is related to these matters. Is $e^{\prime \prime} H \subseteq \widetilde{H}$ of the first category, if in $P M$ $H$ is locally sequentially compact? This is true, if $H=\ell_{2}(D)$ (Brunner [Br86], corollary 2.3.). 
Lemma 24. If in the permutation model $P M \subseteq V(X) A$ is a symmetric linear map whose domain is dense in the Hilbert space $H$ such that in $V(X)$ there is a unique extension $\widetilde{A}$ of $A$ to a self adjoint operator on the completion $\widetilde{H}$ of $H$, then $K=\operatorname{dom} \widetilde{A} \cap P M \in P M$ and the mapping $\widetilde{A} / K: K \rightarrow \widetilde{H} \cap P M$ is in $P M$.

Proof. In $V(X), \widetilde{A}=A^{*}$ and $\operatorname{dom} A^{*}$ with the norm $\|x\|_{1}^{2}=\|x\|^{2}+\|\widetilde{A} x\|^{2}$ is a Hilbert space. $H$ is dense in this space, for otherwise the deficiency indices of $A$ could not vanish ([Du63], p. 1227). The restriction of the new norm to $H$ is in $P M\left[\|x\|_{1}^{2}=\|x\|^{2}+\|A x\|^{2}\right.$ for $\left.x \in H\right]$. Hence in $V(X) \operatorname{dom} \widetilde{A}$ is the completion of the prae-Hilbert space $\left(H,\|\cdot\|_{1}\right)$ of $P M$, whence by the previous lemma $K=\operatorname{dom} \widetilde{A} \cap P M \in P M$.

The mappings $A:\left(H,\|\cdot\|_{1}\right) \rightarrow(H,\|\cdot\|)$ and $\widetilde{A}:\left(\operatorname{dom} \widetilde{A},\|\cdot\|_{1}\right) \rightarrow(\widetilde{H},\|\cdot\|)$ are bounded, whence $\widetilde{A}$ is the unique continuous extension of $A$ from $\underset{\widetilde{A}}{H}$ to $\operatorname{dom} \widetilde{A}$. Since in $P M$ this characterization defines $\widetilde{A} / K: P M \cap \operatorname{dom} \widetilde{A} \rightarrow$ $P M \cap \widetilde{H}, \widetilde{A} / K \in P M$.

e. o. p.

As an application, we investigate intrinsic intrinsic effectiveness. If in $V T$ is a self adjoint operator, such that for some permutation model $P M$ $T=(T \cap P M)^{\sim}$ and in $P M T \cap P M$ is intrinsically effective, then in $P M$ there is some permutation model $Q M$ and a quantum like $A \in Q M$ such that $\widetilde{A}=T \cap P M$. In $V$ there is an $\in$-isomorphic copy $Q M$ ' of $Q M$ which in $V$ is a permutation model ([Br95], theorem 1), whence $T=\left(A^{\prime}\right)^{\sim}$ is intrinsically effective, $A^{\prime} \in Q M^{\prime}$ the quantum like mapping which corresponds to $A$.

For Banach spaces, similar investigations about intrinsically effective operators reveal the noneffective character of the dual spaces, since the permutation model $P M \subseteq V(S)$ satisfies $A C$, iff the dual $X^{*}$ in $P M$ of each $B$-space in $P M$ is dense in the dual in $V(S)$ of the completion $\widetilde{X}$ of $X$. [If $A \in P M$ is an arbitrary set, then $X=\ell_{1}(A)$, formed in $P M$, has the dual $\ell_{\infty}(A)$ in $P M$, which from the outside is dense in $B\left(A, \mathcal{P}^{P M}(A)\right)$ where $B(A, \Sigma)$ is the space of all bounded $\Sigma$-measurable complex valued functions on $A$. Yet $X$ is dense in $\ell_{1}(A)$ of $V(S)$, whose dual is $\ell_{\infty}(A) . B\left(A, \mathcal{P}^{P M}(A)\right)$ is dense in this space, iff $\mathcal{P}^{P M}(A)=\mathcal{P}^{V(S)}(A)$; since $A \in P M$ is arbitrary, $A C$ holds in $\left.P M\right]$. A conversation by one of the authors with Prof. Schachermayer led to the following related result, whose proof we have forgotten.

If $\mathcal{X}$ is an $\aleph_{O}$-categorical countable structure over a finite relational language and $P M \subseteq V(X)$ is generated by Aut $\mathcal{X}$ with the topology of pointwise convergence, then the dual in $V(X)$ of the completion of the space $\ell_{\infty}(X)$ of $P M$ is (isometrically) isomorphic with $\ell_{1}$, iff $\mathcal{X}$ is $\omega$-stable. 


\section{Appendix}

3.1. A philosophical problem. The reference to permutation models in the definition of intrinsic effectivity appears to be redundant, since a Hamiltonian is intrinsically effective, iff it admits a $O N$-base of eigenvectors (proof of lemma 16.) We conclude this paper with a heuristics which explains the rôle of permutation models. To this end we shall relate the FRAENKEL-MOSTOWSKI method to the perception in a wider sense (including the expressive power of the scientific language, also) of an observer, who wants describe the physical world. [Thus observables are compatible, if they can be described by the same observer.] According to BoHR [Bo35] "it lies in the nature of observation, that all experience must ultimately be expressed in terms of classical objects." Hence we may assume, that this world satisfies the laws of classical physics, thereby avoiding a discussion of "quantum reality". Despite their speculative character the investigations of section 3 may serve as a motivation for section 2 .

In order to simplify the discussions about models, we shall require, that if $T$ is an intrinsically effective Hamiltonian on $K$, then each eigenspace of $T$ is assumed to be one dimensional. Moreover, $K$ is assumed to be separable. These requirements are met in most empirically relevant situations.

3.2. Infinite observers. We first investigate the knowledge which is obtained by means of sensual perception. Following KLEENE [Kl56] we identify objects with sets of sensual stimuli. We let the set $S$ represent all possible stimuli. Then $U=\mathcal{P}(S)$ is the set of all objects (i.e. things which cannot be distinguished by means of perception are identified as the same object). An observer $\omega$ is described by the set $S_{\omega} \subseteq S$ of those stimuli which are perceivable for $\omega$ and the set $U_{\omega} \subseteq \mathcal{P}\left(S_{\omega}\right)$ of objects which are perceived and distinguished by $\omega$. Although $X_{\omega}$ is finite for each real observer $\omega$, it is plausible to assume the existence of an infinite set of perceivable objects, too. To this end infinite coalitions of observers are considered which define hypothetical observers $\Omega$ given by $S_{\Omega}=\cup\left\{S_{\omega}: \omega \in \Omega\right\}$ and $U_{\Omega}=\cup\left\{U_{\omega}: \omega \in \Omega\right\}$. [We apply the theory of section 1.5. to the infinite set of names $Y=\cup\left\{\{\omega\} \times X_{\omega}: \omega \in \Omega\right\}$. There $\omega$ corresponds to the order ideal $\mathcal{O}_{\omega}$ for the equivalence relation $R_{\omega}$ on $U \cup\{a\}$ which describes the state of knowledge of the observer $\omega$ : If $x, y \in U$ are objects such that $x \cap S_{\omega}=y \cap S_{\omega} \in U_{\omega}$, then $x R_{\omega} y$, and if $x \cap S_{\omega} \notin U_{\omega}$, then $x R_{\omega} a$. Now $\Omega$ corresponds to the supremum $\mathcal{O}_{\Omega}=\cup\left\{\mathcal{O}_{\omega}: \omega \in \Omega\right\}$.]

We shall identify $\Omega$ with the set $U_{\Omega}$ of objects and we shall assume, that $\Omega$ is an infinite Boolean algebra. If $\Omega$ is the coalition of all possible observers, then stimuli which do not appear in different objects may be identified. Thus $\Omega$ is embedded into $\mathcal{P}(X), X$ the atoms of the algebra $\Omega$.

3.3. Meaningful concepts. An empirical concept is a higher order relation involving mathematical constructions and the elements of an empirical structure $\mathcal{X}$. In order to avoid meaningless concepts which in a set theoretical representation of the concepts might be defined from the $\in$-structure below the elements of 
$\mathcal{X}$, we let these be represented by atoms in the set theoretical sense. We define, that an empirical concept is an element of the structure $V(X), X$ the base set of $\mathcal{X}$.

A relation $R$ on an empirical structure $\mathcal{X}$ is meaningful in the sense of LUCE [Lu78], if it is invariant with respect to all automorphisms of $\mathcal{X}$. A relation $R$ on $\mathcal{X}$ is meaningful relative to a finite set $e \subseteq X$, the base set of $\mathcal{X}$, if it is invariant with respect to all automorphisms which fix $e$. If $\mathcal{X}$ is an $\aleph_{\circ}$-categorical relational structure, this is definability from parameters in $e$. In abstract measurement theory these notions have been extended to higher order relations, i.e. elements of $V(X)$, as follows. A group $(G, \cdot)$ is given. If $d: G \rightarrow S(X)$ is an injective homomorphism into the symmetric group over $X$, then $x \in V(X)$ is meaningful, if $\widehat{d} g(x)=x$ for all automorphisms $g$ of $\mathcal{X}$.

A concept is theoretical, if it is not entirely reducible to perception. Below we shall relate this vague notion to the precise notion of meaningfulness. There are, however, empirical structures which admit meaningless but not theoretical concepts, whence meaningfulness appears to be too restrictive. [If the empirical structure is a Boolean algebra $\Omega$ of perceivable objects, then no $x \in \Omega$ is theoretical, but since a transposition of atoms extends to an automorphism, $x \in \Omega$ is meaningless, unless $x \in\{0,1\}$.]

3.4. Evidently theoretical concepts. If $\mathcal{X}$ is an empirical structure which describes the perception, then we let $P M \subseteq V(X)$ be the permutation model which is generated by $\operatorname{Aut}(\mathcal{X})$ with the product topology of the discrete topology of $X$ and the natural group action. This model is related to the problem of what is a theoretical concept. For we propose, that in the informal definition of a theoretical concept "reducible to perception" implies "meaningfulness relatively to finitely many $x \in X$ ". Moreover, we may assume, that "entirely" means "for all constituents" of a concept. In the set theoretical language this translates to "hereditarily" (c.f. GANDY [Ga80], where mechanisms are represented in a similar way). In view of such assumptions a theoretical concept $x \in V(X)$ cannot be an element of $P M$. This finite support model is typical in the following sense. Up to mutual embeddability of the initial segments (the $\alpha$-th segment consists of all objects of rank $\leq \alpha$ ), the class of finite support models coincides with the class of all permutation models ([Br90], theorems 2.1 and 4.3).

Thus we are lead to identify an idealized observer with a FRAENKELMostowski model $P M \subseteq V(X)$. The model $P M$ is related to the perception of the idealized observer $P M$ by means of the following definition: A concept $x \in V(X)$ is evidently theoretical for the observer $P M$, if $x \notin P M$. In the case of the above finite support model, this appears to be a sufficient condition for a concept to be theoretical (intuitively, a theoretical concept is evidently theoretical, if it is empirically meaningless for formal reasons already). In the general case we postulate sufficiency. In order to relate the abstract model to the real world, we then need to specify a correspondence rule, for otherwise the condition, that $x \in V$ is not evidently theoretical does not represent any restriction, since each concept of the $Z F C$ universe $V$ may be reconstructed 
within $V(\varnothing) \subseteq P M$. In celestial mechanics the notion of evidently theoretical concepts may be traced back to J. KEPLER's "non-entia" in Harmonices mundi libri $V$ (Linz 1619), where it is argued, that empirically realizable concepts correspond to constructions by means of ruler and compass.

As an example, let us consider a coalition $\Omega$ whose language of concept formulation permits for each newly formed concept the use of finitely many objects only in characterizing its constituent concepts. As in section $3.2 X$ is the set of the atoms of $\Omega$ and $\Omega$ is represented by a set algebra $X_{\Omega} \subseteq \mathcal{P}(A)$, $A$ the atoms of $V(X)$. If the concept $x \in V(X)$ depends on a finite set $e \subseteq \Omega$ of objects, then $x$ is meaningful relative to $e$ in the structure $\Omega$ (i.e. $(\widehat{d} g)(x)=x$, if $g \in \operatorname{Aut}(\Omega)$ fixes $e$.) The concepts $x \in V(X)$ which satisfy this condition hereditarily are the elements of the WEGLORZ model $P M_{\Omega}$. (In terms of the empirical structure $\mathcal{X}=\Omega$, the finite support model of the previous paragraph is $P M_{\Omega}(\Omega)$ in the notation of $[\mathrm{Br} 95]$.)

In view of the proofs of section 2 we need to correlate $F M$ to a specific type of observation. We consider nerve nets with an infinite past over the language $L=\{0,1\}$. Then a set $W \subseteq L^{<\omega}$ of finite sequences can be recognized, iff it is definite (KLEENE [Kl56]: there is a finite set $E \subseteq \omega$, such that for all words $w_{i} \in L^{n}, n \in \omega$, such that $w_{0}\left|E=w_{1}\right| E, w_{0} \in W$ and $w_{1} \in W$ are equivalent; i.e. $W$ depends on $E$.) We also assume, that besides the objects (definite sets) the observer perceives the complexity of the input by means of the relations $x R_{n} y$ iff $x(n)=y(n)$ and $n \in \operatorname{dom} x=\operatorname{dom} y$. Then the group of automorphisms of this structure is $\mathbb{Z}_{2}^{\omega}$ with the group action $d$, $(d g)(f)(n)=g(n)+f(n)$ modulo 2 for $n \in \operatorname{dom} f$. Its topology is generated by stab $x=\{g: g / E=0\}$ where $x$ is a definite set which depends on $E$. It is the product topology. The corresponding model $P M$ is closely related to $F M$, since it is generated by the same topological group $G$ as $F M$ (but with a different group action), whence the initial segments of $F M$ can be $\widetilde{\epsilon}$ isomorphically embedded into $P M$ and vice versa.

3.5. Correspondence rules. We let the Hamiltonian $T$ on $K$ be intrinsically effective. Since $K$ is separable, in $F M$ there is a Hilbert space $H$ with a quantum like mapping $A$ on $H$ such that in $V T U=U \widetilde{A}$ for some bijective isometry $U: \widetilde{H} \rightarrow K$ (lemma 16). As is suggested by the preceding discussion, $F M$ may be identified with an (idealized) observer who investigates the Schrödinger equation $T \sigma=i \cdot \frac{\partial \sigma}{\partial t}$. A correspondence rule between the empirical reality (i.e. quantum theory on $K$ ) and its reconstruction in terms of perception (i.e. finitary quantum theory on $H$ ) is given by the isometry $U$.

Modulo this heuristics, a pure state $\sigma(0) \in K$ corresponds to a state preparation procedure which can be described without a reference to evidently theoretical concepts, if $U^{-1} \sigma(0) \in \widetilde{H} \cap F M$. As $F M$ satisfies $M C, \widetilde{H} \cap F M=H$ (lemma 23), whence $\sigma(0) \in U^{\prime \prime} H=\operatorname{dom} T^{\circ}$ (because the eigenspaces of $T$ are finite dimensional) and $U^{-1} T^{\circ} U \in F M$ (lemma 24). Therefore both the Hamiltonian $T$ and the state evolution may be reconstructed within $F M$ (section 2.6), $U^{-1} \sigma(t) \in H \in F M$, whence the computation of $\sigma(t)$ does not depend 
on evidently theoretical concepts. Moreover if $S$ is intrinsically effective and commeasurable with $T$, then $S=f(T)$ for some Borel function $f$ [since the eigenspaces of $T$ are one dimensional, this follows from the proof of lemma 20 ] and the expected value of the measurement $S$ of $\sigma(t)$ may be determined without the use of evidently theoretical concepts, even if $\sigma(0)$ depends on such concepts; $<S \sigma(t), \sigma(t)>=\operatorname{tr}(V(-t) f(A) V(t) B)$ for some mixture $B \in F M$ and $V(t)=\exp (-i \cdot A \cdot t)$. (If the eigenspaces of $T$ are finite dimensional but not nececessarily one dimensional, then the same conclusion holds with a different correspondence rule.)

$F M$ therefore supports the intuition, that given a Hamiltonian $T$, a state preparation procedure which defines a pure state $\sigma(0)$ is a meaningful input for $T$ (i.e. the resulting experimental configuration is not theoretical), iff $\sigma(0) \in \operatorname{dom} T^{\circ}$. Thus $T$ is intrinsically effective, iff it is determined by the meaningful inputs [i.e. dom $T^{\circ}$ is dense.] In general, however, this intuition is false. In the model $V(X)$ no state is evidently theoretical, but $\operatorname{dom} T^{\circ}$ may contain too few states to determine $T$ (lemma 6). We therefore restrict such a heuristics to $F M$ and interpret intrinsically effective quantum theory as that fragment of constructive quantum mechanics which is compatible with the existence of RuSSELL's socks.

3.6. Conclusion. The theory of intrinsic effectivity is an alternative approach to answer questions of constructivity within $Z F C$. It corresponds to a version of effective mathematics, that replaces the axiom of choice by the multiple choice axiom.

The classical approach is the theory of abstract computability of POUREL and RiCHARDS [Po89] which focuses on the bounded self adjoint operators on separable Hilbert spaces. Therefore its applicability as a tool for the working physicist might be restricted somewhat (but c.f. [Ba93] and [Sc $\infty]$ ), for as HeLLMAN [He93], p. 240, remarks, unbounded operators are needed even for the most elementary textbook problems: "Moreover, typically such operators are defined on an orthonormal basis (e.g. eigenfunctions of the Hamiltonian) which at least in standard problems presumably are amenable to a constructive treatment". Since such operators (e.g. the harmonic oscillator which is HELLMAN's standard example) are intrinsically effective, our results verify HeLLMAN's "presumably".

The Hamiltonians of the hydrogen atom and the helium atom have continuous spectra and therefore evade an analysis in terms of intrinsic effectivity. [This concerns scattering phenomena. The computations in chemistry of energy levels of electron orbits depend on intrinsically effective direct summands, only.] Moreover, some fundamental theorems of quantum theory, such as the GLEASON-MAEDA characterization of the states neither are constructive nor effective. We therefore again arrive at HELLMAN's conclusion (p. 243), that "the quantum world is indeed a hostile environment for many species of constructivist mathematics".

We conjecture, that this difficulty might be due to the implications of the constructive analysis of quantum mechanics for the problem of empirical 
realizability (c.f. E.P. Wigner in Z. Phys. 33(1952), p. 101.) Such speculations, however, transcend the scope of the present paper. The interpretation of the extra physical notions of our paper in terms of the perceptions of observers indicates the rôle of intrinsic effectivity in this context.

Concerning the status of the realizability problem, let us just mention, that if thought experiments are applied to observables of quantum theory which are not empirically realizable, then dubious conclusions may be drawn. An example of incorrect reasoning is VON NEUMANN's refutation of a hidden parameter theory also for the spin $\frac{1}{2}$ particle (c.f. BELL's rebuttal [Be66]) ${ }^{4}$. Such concerns over the proper meaning of quantum mechanics have produced a significant body of literature, reviewed in JAMMER [Ja74], which illustrates, that there is a gap in our knowledge of which mathematical observables are empirically realizable.

\section{References}

[Al86] S. Albeverio, J. E. Fenstad, J. E. Hoegh-Krohn and T. LindStrom: "Nonstandard Methods in Stochastic Analysis and Mathematical Physics", Academic Press, New York (1986).

[Ba93] M. BAAz, N. Brunner and K. SvozIL: "Interpretations of Combinatory Algebras", in J. Czermak: "Philosophy of Mathematics", HPT, Wien (1993), 393-406.

[Be66] J. S. BeLL: "On the Problem of Hidden Variables in Quantum Mechanics", Reviews of Modern Physics 38(1966), 447-452.

[Be76] P. A. Benioff: "Models of Zermelo Fraenkel Set Theory as Carriers for the Mathematics of Physics. I", Journal of Mathematical Physics 17(1976), 618-628.

[Bo35] N. Bohr: "Can Quantum-Mechanical Descriptions of Physical Reality be Considered Complete?", Physical Reviews 48(1935), 696-702.

[Bi36] G. Birkhoff and J. von Neumann: "The Logic of Quantum Mechanics", Annals of Math. 37(1936), 823-843.

[Bo88] W. Boos: Abstract, The Journal of Symbolic Logic 53 (1988), 1289.

[Br95] N. Brunner: "A Modal Logic of Consistency", Rendiconti Sem. Mat. Univ. Padova 93(1995).

[Br90] N. Brunner: "The Fraenkel-Mostowski Method, Revisited", Notre Dame J. Formal Logic 31(1990), 64-75.

[Br86] N. Brunner: "Linear Operators and Dedekind Sets", Math. Japonica 31(1986), 1-16.

\footnotetext{
4 There are negative results due to Kochen and SPECKER [Ko67], that hidden variable theories do satisfy a different functional calculus (dependency on parameters encoding the experimental context) and due to BELL, that hidden variable theories are not local. BoOs [Bo88] defines hidden variables for a ground model of set theory in some generic extension, thereby retaining a strong functional calculus. The theory of PITOWSKY [Pi89] is both almost noncontextual (the lattice operations are preserved almost surely) and local, but the axioms of probability theory are weakened.
} 
[Br83] N. Brunner: "The Axiom of Choice in Topology", Notre Dame J. Formal Logic 24(1983), 305-317.

[Br83] N. Brunner: "Kategoriesätze und multiples Auswahlaxiom", Zeitschrift f. math. Logik u. G. d. Mathematik (Math. Logic Quarterly) 29(1983), $435-443$.

[Br82] N. Brunner: "Dedekind-Endlichkeit und Wohlordenbarkeit", Monatshefte f. Math. 94(1982), 9-31.

[Du57] N. Dunford and J. T. SchwARTZ: "Linear Operators I", Interscience Pure and Appl. Math. 7, New York (1957).

[Du63] N. Dunford and J. T. Schwartz: "Linear Operators II", Interscience Pure and Appl. Math. 7, New York (1963).

[Ga80] R. O. Gandy: "Church's Thesis and Principles for Mechanisms", in J. BARWise et al.: "The Kleene Symposium", North Holland Studies in Logic 105, Amsterdam (1980), 123-148.

[Ha82] P. R. Halmos: "A Hilbert Space Problem Book," Springer Graduate Texts in Math. 19, Berlin (1982).

[He93] G. Hellmann: "Constructive Mathematics and Quantum Mechanics: Unbounded Operators and the Spectral Theorem," J. Philosophical Logic 22 (1993), 221-248.

[Hi84] N. Hindman and P. Milnes: "The Ideal Structure of $X^{X}$ ", Semigroup Forum 30(1984), 41-51.

[Is89] H. IshiharA: "On the Constructive Hahn-Banach theorem", Bulletin London Math. Soc. 21(1989), 79-81.

[Ja74] M. JAMmeR: "The Philosophy of Quantum Mechanics", Interscience, New York (1974).

[Ja68] J. M. JAUCH: "Foundations of Quantum Mechanics", Addison-Wesley, Reading (1968).

[Je73] T. JECH: "The Axiom of Choice," North Holland Studies in Logic 75, Amsterdam (1973).

[Ka54] I. Kaplansky: "Infinite Abelian Groups," M.U.P., Ann Arbor (1954).

[K156] S. C. KLEEnE: "Representation of Events by Nerve Nets", in C. E. ShanNON et al.: "Automata Studies", P. U. P. Annals of Math. Studies 34, Princeton (1956), 3-41.

[Ko67] S. Kochen and E. P. SPECKer: "The Problem of Hidden Variables in Quantum Mechanics", J. Math. Mech. (Indiana Univ. J.) 17 (1967), 5987.

[Ko65] S. Kochen and E. P. SPeCKer: "Logical Structures Arising in Quantum Theory", in J. AdDison et al.: "The Theory of Models", North Holland Studies in Logic 55, Amsterdam (1965).

[La62] H. LÄUChli: "Auswahlaxiom in der Algebra", Comm. Math. Helvetii $37(1962), 1-18$. 
[Lu78] J. D. LuCE: "Dimensionally Invariant Numerical Laws Correspond to Meaningful Qualitative Relations", Philosophy of Science 45(1978), 1-16 .

[Ma80] S. MaedA: "Lattice Theory and Quantum Logic", Makishoten, Tokyo (1980).

[Mo91] M. Morillon: "Duaux Continus et Axiome du Choix", Seminaire d' Analyse (Clermont-Ferrand), 1991.

[vN29] J. von Neumann: "Allgemeine Eigenwerttheorie Hermitescher Funktionaloperatoren", Mathematische Annalen 102 (1929), 49-131.

[Pe93] A. Peres: "Quantum theory. Concepts and methods", Kluwer, Dordrecht (1993).

[Pe78] A. PelC: "On some Weak Forms of the Axiom of Choice in Set Theory", Bull. Acad. Polon. Sc. Ser. Math. 26(1978), 585-589.

[Pi89] I. Pitowsky: "Quantum Probability - Quantum Logic", Springer Lecture Notes in Physics 321, Berlin (1989).

[Pi72] D. Pincus: "Independence of the Prime Ideal Theorem from the Hahn Banach Theorem", Bulletin American Math. Society 78(1972), 766- 770.

[Po89] M. B. PourEl and I. Richards: "Computability in Analysis and Physics", Springer Perspectives in Math. Logic, Berlin (1989).

[Ra73] H. Radjavi and P. Rosenthal: "Invariant Subspaces", Springer Ergebnisse d. Math. 77, Berlin (1973).

[Re94] M. Reck, A. Zeilinger, H. J. Bernstein and B. Bertani: "Experimental Realization of any Discrete Unitary Operator", Phys. Rev. Letters 73(1994), 58-61.

[Ru85] H. Rubin and J. E. Rubin: "Equivalents of the Axiom of Choice, IF", North Holland Studies in Logic 116, Amsterdam (1985).

[Sa75] G. SageEv: "An Independence Result Concerning the Axiom of Choice", Annals Math. Logic 8 (1976), 1-184.

[Sc $\infty$ ] M. Schaller and K. SvozIL: "Partition Logics of Automata", Nuovo Cimento (to appear).

[Ti62] E. C. Titchmarsh:"Eigenfunction Expansions Associated with Second Order Differential Equations I", Oxford U. P., London (1962).

[Vo79] P. VopênKa: "Mathematics in the Alternative Set Theory", Teubner, Leipzig (1979).

[Wg69] B. Weglorz: "A Model of Set Theory $\mathcal{S}$ over a Given Boolean Algebra", Bull. Acad. Polon. Sc. Ser. Math. 17 (1969), 201- 202. 